\title{
Discussion on the spectral coherence between planetary, solar and climate oscillations: a reply to some critiques
}

\author{
Nicola Scafetta ${ }^{1,2}$ \\ the date of receipt and acceptance should be inserted later
}

\begin{abstract}
During the last few years a number of works have proposed that planetary harmonics regulate solar oscillations. Also the Earth's climate seems to present a signature of multiple astronomical harmonics. Herein I address some critiques claiming that planetary harmonics would not appear in the data. I will show that careful and improved analysis of the available data do support the planetary theory of solar and climate variation also in the critiqued cases. In particular, I show that: (1) high-resolution cosmogenic ${ }^{10} \mathrm{Be}$ and ${ }^{14} \mathrm{C}$ solar activity proxy records both during the Holocene and during the Marine Interglacial Stage 9.3 (MIS 9.3), 325-336 kyear ago, present four common spectral peaks (confidence level $\gtrsim 95 \%$ ) at about 103, 115, 130 and 150 years (this is the frequency band that generates Maunder and Dalton like grand solar minima) that can be deduced from a simple solar model based on a generic non-linear coupling between planetary and solar harmonics; (2) time-frequency analysis and advanced minimum variance distortion-less response (MVDR) magnitude squared coherence analysis confirm the existence of persistent astronomical harmonics in the climate records at the decadal and multidecadal scales when used with an appropriate window length $(L \approx 110$ years) to guarantee a sufficient spectral resolution to solve at least the major astronomical harmonics. The optimum theoretical window length deducible from astronomical considerations alone is, however, $L \gtrsim 178.4$ years because the planetary frequencies are harmonics of such a period. However, this length is larger than the available 164-year temperature signal. Thus, the best coherence test can be currently made only using a single window as long as the temperature instrumental record and comparing directly the temperature and astronomical spectra as done in Scafetta (2010) and reconfirmed here. The existence of a spectral coherence between planetary, solar and climatic oscillations is confirmed at the following periods: 5.2 year, 5.93 year, 6.62 year, 7.42 year, 9.1 year (main lunar tidal cycle), 10.4 year (related to the 9.93-10.87-11.86 year solar cycle harmonics), 13.8-15.0 year, 20 year, 30 year and 61 year, 103 year, 115 year, 130 year, 150 year and about 1000 year. This work responds to the critiques of Cauquoin et al. (2014), who ignored alternative planetary theories of solar variations, and of Holm (2014a), who used inadequate physical and time frequency analysis of the data.
\end{abstract}

Cite as: Scafetta, N., 2014. Discussion on the spectral coherence between planetary, solar and climate oscillations: a reply to some critiques. Astrophys Space Sci 354, 275-299, 2014. DOI: 10.1007/s10509-014-2111-8

\footnotetext{
${ }^{1}$ Meteorological Observatory, Department of Earth Sciences, Environment and Georesources, University of Naples Federico II, Largo S. Marcellino 10, 80138 Naples, Italy. ${ }^{2}$ Active Cavity Radiometer Irradiance Monitor (ACRIM) Lab, Coronado, CA 92118, USA. ${ }^{3}$ Duke University, Durham, NC 27708, USA. +1(919) 225-7799; nicola.scafetta@unina.it, nicola.scafetta@gmail.com
} 


\section{Introduction}

Wolf (1859) proposed that solar variability could be modulated by the combined effect of the planets, in particular by Venus, Earth, Jupiter and Saturn. However, solar scientists have been skeptical about the possibility of a planetary theory of solar variation because according Newtonian classical gravitational physics the planets appear to be too far from the Sun to effectively influence its activity. For example, Newtonian tidal accelerations induced by the planets on the Sun's tachocline appear to be too small (e.g.: Callebaut et al., 2012; Scafetta, 2012d). However, Newtonian gravitational physics alone does not explain how the Sun and the heliosphere work because, as well known, electromagnetism, quantummechanics and nuclear fusion physics are required as well (Bennett et al., 2014).

Strong internal nuclear fusion amplification tidal mechanisms (Scafetta, 2012d; Wolff and Patrone, 2010), torques acting on a non-spherical tachocline (Abreu et al., 2012) and electromagnetic coupling throughout the heliosphere (e.g.: Scafetta and Willson, 2013b) have been proposed as possible mechanisms.

Evidences for a planet-induced stellar activity have been noted in numerous stars, in particular when the phenomenon becomes macroscopic due to the presence of a hot Jupiter (Scharf, 2010; Shkolnik et a., 2003; Shkolnik et al., 2005). Wright et al. (2008) detected a quasi 9-year activity cycle in a star with a Jupiter-like planet on a 9.2-year circular orbit with radius $4.2 \mathrm{AU}$.

In the case of our Sun, if its activity is regulated by planetary harmonics, the problem is expected to be quite subtle. Multiple planetary harmonics would produce complex beats with the solar internal cycles, and solar activity would not mirror just a single and easily recognizable planetary harmonic (e.g. the 11.86-year Jupiter orbital cycle) but would non-linearly respond to a complex synchronization pattern emerging from the harmonics of the entire solar system (e.g.: Jakubcová and Pick, 1986; Scafetta, 2014).

Although the physical problem is evidently still open, numerous recent works have promoted the theory by claiming that solar activity presents empirical signatures of planetary harmonics from the monthly to the millennial time scales (e.g.: Abreu et al., 2012; Charvátová, 2009; Fairbridge and Shirlev, 1987; Iose,, 1965; McCracken et al., 2013, 2014; Scafetta, 2012c) d; Scafetta and Willson, 2013b; Sharp, 2013; Shirlev et al., 1990; Tan and Cheng, 2013). Because the Earth's climate appears closely linked to solar variations (e.g.: Hoyt and Schatten, 1997; Steinhilber et al., 2012, and many others), similar harmonics have also been found in the climate system at multiple decadal, secular and millennial time scales (e.g.: Abreu et al., 2012; Scafetta, 2010, 2013b). Aurora, sunspot, and meteorite fall records too present planetary harmonics and suggest gravitational, electromagnetic and luminosity links between astronomical and climatic records (e.g.: Scafetta, 2012a; Scafetta and Willson, 2013a).

Spectral coherence between astronomical and solar records was found from the monthly to the millennial time scales (e.g.: Scafetta, 2010, 2012c, 2013b, and others) and, as Wolf (1859) conjectured, also the very 11-year Schwabe solar cycle appears mostly synchronized to the harmonics generated by the orbital revolution of Venus, Earth, Jupiter and Saturn (e.g.: Hung, 2007; Salvador, 2013; Scafetta, 2012c d; Wilson, 2013). A special issue collecting works on "pattern in solar variability, their planetary origin and terrestrial impacts" has been recently published (Mörner et al., 2013; Scafetta, 2014, and other authors). 
However, critiques questioning some of the above claims have also appeared. Herein I will focus on the critiques by Cauquoin et al. (2014) and Holm (2014a).

Cauquoin et al. (2014) critiqued Abreu et al. (2012) arguing that if the solar-planetary harmonic coherence highlighted by Abreu et al. (2012) in several ${ }^{14} \mathrm{C}$ and ${ }^{10} \mathrm{Be}$ series throughout the Holocene period (Steinhilber et al., 2012) were real, similar harmonics had to be found also in "the record of ${ }^{10} \mathrm{Be}$ in the EPICA (European Project for Ice Coring in Antarctica) Dome C (EDC) ice core from Antarctica during the Marine Interglacial Stage 9.3 (MIS 9.3), 325-336 kyear ago". About the secular scales Cauquoin et al. (2014) claimed to have found only a very "limited similarity with the periodicities ... predicted by the planetary tidal model of Abreu et al. (2012)."

Holm (2014a) critiqued Scafetta (2010) by arguing that on the decadal and multidecadal scales time-frequency analysis based on $L=60$ year windows and magnitude squared coherence analysis based on $L=20$ and 30 year windows would show time-varying spectral lines that look "very different from the nearly constant lines in the time-frequency plot for the speed of the center of mass of the solar system (SCMSS)." Both critical studies were interpreted as questioning the planetary theory of solar and Earth's climate variability.

However, in addition to Scafetta's findings several solar and climatic oscillations have been identified throughout the Holocene. These oscillations can be easily associated with many well-known astronomical harmonics. Pustil'nik and Din (2004) found an influence of the 11-year solar activity cycle on the state of the wheat market since medieval England. Chylek et al. (2011) found evidences for a prominent near 20-year oscillation in multisecular ice-core records. 50 to 70-year climatic oscillations have been discovered in numerous climatic instrumental and proxy records (e.g.: Agnihotri and Dutta, 2003; Davis and Bohling, 2001; Ievreieva et al., 2008; Klvashtorin et a., 2009; Knudsen et al., 2011; Loehle and Scafetta, 2011; Mazzarella and Scafetta, 2012; Oian and Lu, 2010; Scafetta, 2010; Scafetta et al.,2013, and many others). Oian and Lu (2010); Puetz et al. (2014); Scafetta (2012c) found evidences for a quasi 115-year climatic oscillation in millennial multiproxy climatic records. A quasi 900-1000 year oscillation has also been observed in numerous climatic records throughout the Holocene (Bond et al., 2001; Christiansen and Ljungqvist, 2012; Kerr, 2001; Scafetta, 2012a). Similar oscillations are found among proxy records of solar activity, in the aurora records and in the oscillations of the heliosphere (e.g.: Ogurtsov et al., 2002; Iakubcová and Pick, 1986; Scafetta, 2012a; (Scafetta and Willson, 2013b). Another set of climatic oscillations appear to be lunar related such as the 8.85-9.3 year oscillation found in the Atlantic Multidecadal Oscillation and in the Pacific Decadal Oscillation indexes (c.f.: Scafetta, 2012b; Manzi et al., 2012). Additional Soli-lunar harmonics are present too and interfere with each other generating the El Niño-Southern Oscillation-like inter-annual variation (cf. Wang et al., 2012).

In this paper I comment and refute Cauquoin et al. (2014) and Holm (2014a)'s critiques, and I upgrade my previous analysis (Scafetta, 2010) with more advanced methodologies and arguments. The main astronomical periods of interest in this work are at about 5.2 years, 5.93 years, 6.62 years, 7.42 years, 9.1 years (main average solar-lunar tide cycle), 10.4 years (related to the 9.93-10.87-11.86 year solar cycle harmonics), 13.8 years, $\sim 20$ years, $\sim 30$ years and $\sim 61$ years, 103 years, 115 years, 130 years, 150 years and quasi 1000 years (cf.: Scafetta, 2010, 2012a) d, 2014). Other planetary astronomical periods in solar records have been found, e.g. numerous sub annual oscillations (e.g.: Shirlev et al., 1990; Scafetta and Willson, 2013b a; Tan and Cheng, 2013), the $\sim 87$ and $\sim 207$ year oscillations (e.g.: Abreu et al., 2012; 
Bond et al., 2001; Scafetta, 2012c; Scafetta and Willson, 2013a) and others, but these oscillations are not discussed in detail here because they were not analyzed in the critiqued works.

\section{Common evidences for a planetary influence on solar activity 330,000 years ago and during the Holocene}

Cauquoin et al. (2014) analyzed a record of ${ }^{10} \mathrm{Be}$ in the EPICA (European Project for Ice Coring in Antarctica) Dome C (EDC) ice core from Antarctica during the Marine Interglacial Stage 9.3 (MIS 9.3), referring to 325-336 kyear ago. Their ${ }^{10} \mathrm{Be}$ records are shown in Figure 1A and their Fourier spectra are shown in Figure 1B-C. According Cauquoin et al. (2014) own figures, their ${ }^{10} \mathrm{Be}$ concentration and flux records are quite noisy and likely effected by a low frequency component that they detrend with a filtering: see Figure 1A. Only four secular frequencies were found at about 103 year (99\% confidence), 115 year ( $\approx 95 \%$ confidence), 130 year $(99 \%$ confidence) and 150 year ( $\approx 95 \%$ confidence). Note that Cauquoin et al. (2014) highlighted with dash black lines only the spectral peaks at the 99\% confidence level ignoring the other two spectral peaks that can be discerned about at the 95\% confidence level curve. In Figure 1 I highlighted with red lines the $95 \%$ confidence spectral peaks.

By taking into account these four spectral peaks the spectral analyses among the three studied records are consistent to each other: compare Figure 1B, 1C and 1D. The 95\% confidence level is typically used in the scientific literature when Fourier or wavelet spectral analysis are adopted (e.g.: Ghil et al.,2002; Knudsen et al., 2011, http: / / web.atmos.ucla.edu/tcd/ / ssa/ ; http://duducosmos.github.io/PIWavelet/).

Cauquoin et al. (2014) questioned the planetary theory of solar variation because they claimed a "very limited similarity between the periodicities in this record compared to those found in a proxy record of solar variability during the Holocene (Steinhilber et al., 2012), or those predicted by the planetary tidal model of Abreu et al. (2012)." However, it is evident that Cauquoin et al. (2014) could not make any definitive conclusion about periodicities shorted than 100 years and larger than 150 years because according their own confidence level estimates their records do not have sufficient statistical power in those time scales, as evident in their own figures reproduced herein in Figures 1B and 1C.

Cauquoin et al. (2014) also showed wavelet analysis diagrams using the methodology proposed by Grinsted et al. (2004). These diagrams show an intermittent patterns. However, wavelet analysis does not substitute Fourier analysis. Wavelets use small sub-windows whose length is a certain multiple of the analyzed spectral period, known also as scale. Because the left and right border confidence lines used in the wavelet diagrams of Cauquoin et al. (2014) at period of 1000 years are about 1500-year wide, the effective wavelet length was about 3 times the spectral period. As explained in Section 3.4, to separate close harmonics with spectral analysis methodologies, which include also wavelet based methodologies, there is a need of using segments longer than the beat period among contiguous harmonics. The contiguous beat periods among harmonics with periods of 103,115, 130 and 150 years are 987,996 and 975 years respectively (Eq.5). Thus, even if the records were error-free, which is unrealistic, Cauquoin et al. (2014) wavelet analysis can not separate the 4 secular harmonics because the minimum required window length is 10 times the spectral period while the length of typical wavelets such as the Morlet or Mexican Hat and others is up to about 3 times the analyzed scale, 

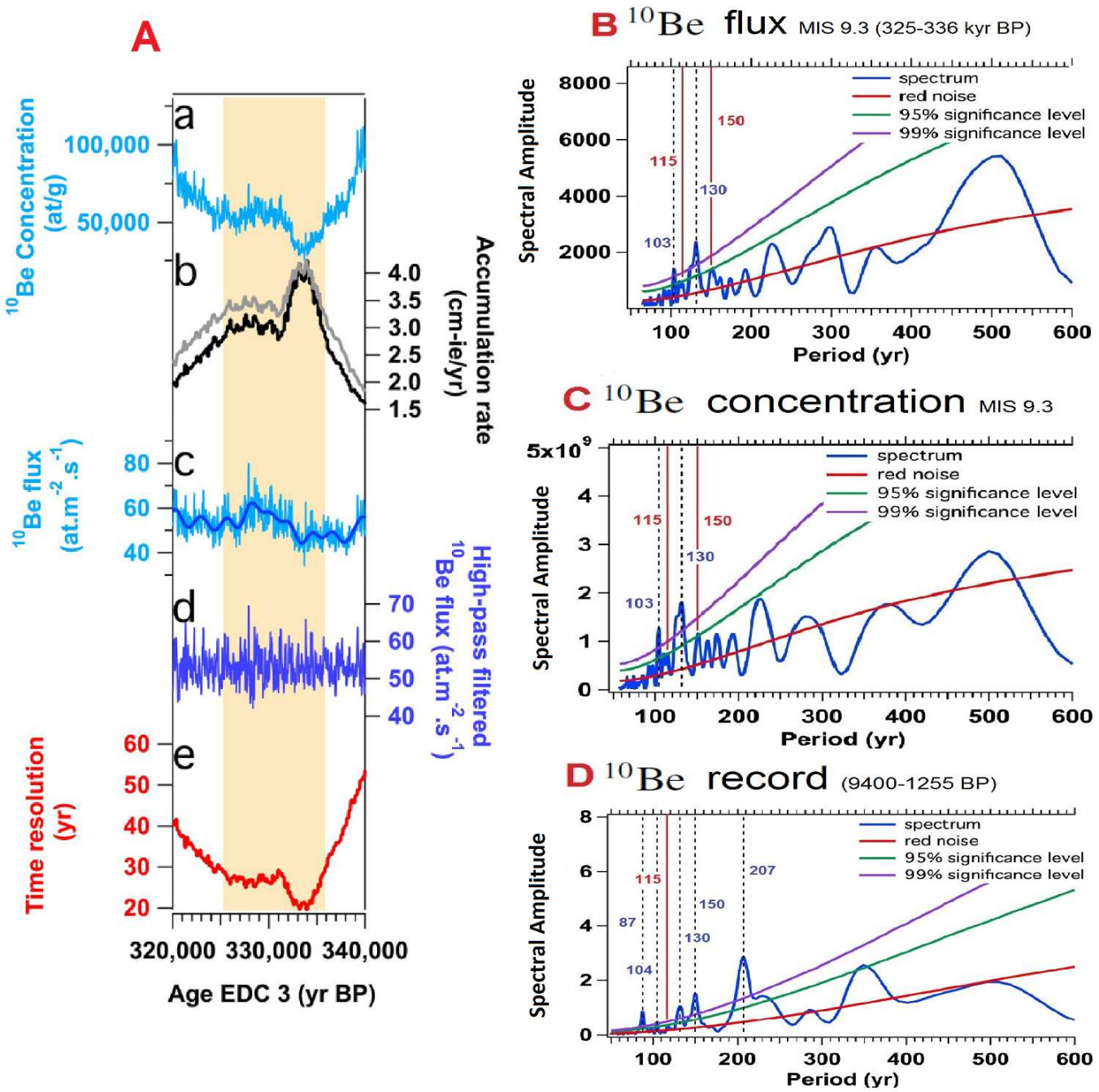

Fig. 1 [A] ${ }^{10} B e$ concentration (top); $\delta D$ and ice accumulation rate; ${ }^{10} B e$ flux; 2000 -year high-band passed ${ }^{10} B e$ flux; and ${ }^{10} B e$ sample resolution in EDC ice core (bottom). [B, C, D] Fourier spectrum analysis of the ${ }^{10} \mathrm{Be}$ flux record at EDC during the interglacial period MIS 9.3 (325-336 kyear), of the ${ }^{10} \mathrm{Be}$ concentration profile during MIS 9.3 and of the composite from Steinhilber et al. (2012) during the Holocene. The figures are adapted from Cauquoin et al. (2014) and highlight 4 main common frequencies at about 103, 115, 130 and 150 years with a statistical confidence of 99\% (blue) and 95\% (red). The Fourier analyses and their confidence error estimates are based on Schulz and Mudelsee (2002).

as implicit in Cauquoin et al. (2014) figures. Thus, Cauquoin et al. (2014) wavelet analysis can only highlight the mutual beats among the four frequencies of interest and show intermittent patterns, as their figures depict.

Figure 1D shows that the Holocene solar proxy model by Steinhilber et al. (2012) also presents spectral peaks at about 104 year (99\% confidence), 115 year ( 95\% confidence), 130 year (99\% confidence) and 150 year (99\% confidence) (Abreu et al., 2012; Cauquoin et al., 2014; Scafetta and Willson, 2013a). The model of Abreu et al. (2012), which was based on the planetary torque on the Sun's tachocline, was able only to highlight peaks at about 104 and 150 years. Poluianov and Usoskin (2014) showed that these peaks could be aliasing artifacts caused by the calculation algorithm that was based on an annual average while three planets (Mercury, Venus and Earth) have orbital periods $\leq 1$ year. In any case, Abreu et al. (2012) planetary model could explain at most two spectral peaks (at about 103-104 and 150 year periods), but could not explain the other two observed spectral peaks (at about 115 and 130-year periods). 
However, Cauquoin et al. (2014) did not realize that the alternative planetary model proposed by Scafetta (2012c), which was based on the combination of the tidal harmonics of Jupiter and Saturn modulating the 11-year solar cycle plus a non-linear solar and geophysical response producing the final analyzed nucleotide output records. As shown below, Scafetta's model predicts major solar harmonics at about 115 and 130 years and the other harmonics of interest.

As briefly summarized in the Appendix, Scafetta (2012c, 2014) noted that spectral analysis of the sunspot records since 1700 reveals that the Schwabe solar cycle is characterized by three main spectral peaks at about 9.93, 10.87 and 11.86 years: see Figure 2A. The spectral peak at 9.93 year is coherent to the spring tidal harmonic generated by Jupiter and Saturn $\left(P_{J S}=0.5 * P_{J} P_{S} /\left(P_{S}-P_{J}\right)=9.93\right.$ year, where $P_{J}=11.86$ years and $P_{S}=29.45$ years are the orbital periods of Jupiter and Saturn, respectively), while the 11.86-year spectral peak is coherent with the tidal harmonic generated by the orbital period of Jupiter. The central 10.87 year harmonic is the central Schwabe solar cycle period (cf. Pustil'nik and Din, 2004). The central $\sim 10.87$ harmonic may be generated by the solar dynamo responding non-linearly to planetary harmonics (e.g.: Hung, 2007; Scafetta, 2012d; Wilson, 2013).

This result suggests that the sunspot cycle is modulated by the two Jupiter-Saturn planetary tidal forces. Scafetta (2012d) proposed a physical model to explain how these tides could effect solar luminosity, but alternative electromagnetic mechanisms may be present too and should be characterized by similar and/or related harmonics.

Scafetta (2012c) constructed a simple three-frequency solar model that uses as input a simple harmonic function made: (1) of the sum of the three harmonics found in the sunspot record modulated by their own millennial beat harmonic; (2) of the astronomically deduced phases of the tidal harmonics generated by Jupiter and Saturn; and (3) on the sunspot number record from 1750 to 2010. The Sun is then assumed to process the input harmonic function via numerous internal mechanisms that are very reasonably non-linear. See the Appendix for a summary of the equations of the model.

The final output functions, that is what is measured and analyzed such as the ${ }^{14} \mathrm{C}$ and ${ }^{10} \mathrm{Be}$ records, are non-linear modification of the input three-frequency harmonic function. As well know by simple algebraic relation, non-linear processing of a harmonic function generates additional frequencies associated to the mutual beats and sub-harmonics of the input generating frequencies. For example, if an input function made of two harmonics as $f(t)=\cos \left(2 \pi f_{1} t\right)+$ $\cos \left(2 \pi f_{2} t\right)$ is non-linearly processed into the function $g(t)=(1+f(t))^{2}$, the latter is characterized by 6 harmonics: $f_{1}$, $f_{2}, 2 f_{1}, 2 f_{2},\left|f_{1}+f_{2}\right|$ and $\left|f_{1}-f_{2}\right|$.

More complex non-linearizations of an input harmonic function would produce more harmonics but these are always related to the same mutual beats and sub-harmonics of the generating frequencies. Thus, any generic non-linearization of the input three-frequency model would generate a similar set of theoretical expected frequencies. Therefore, there is no need to know the exact physical functions of the internal solar processing mechanisms to determine the expected frequencies that can emerge when a non-linear system is forced by a harmonic forcing.

Because the sunspot number record presents minima that converge to about zero, which indicates the existence of some activation threshold and other non-linearities in the solar internal mechanisms, Scafetta (20120) schematically represented the process by generating an output signal where the negative values of the input original harmonic sequence are set to zero (see Figure 2E and the Appendix). 

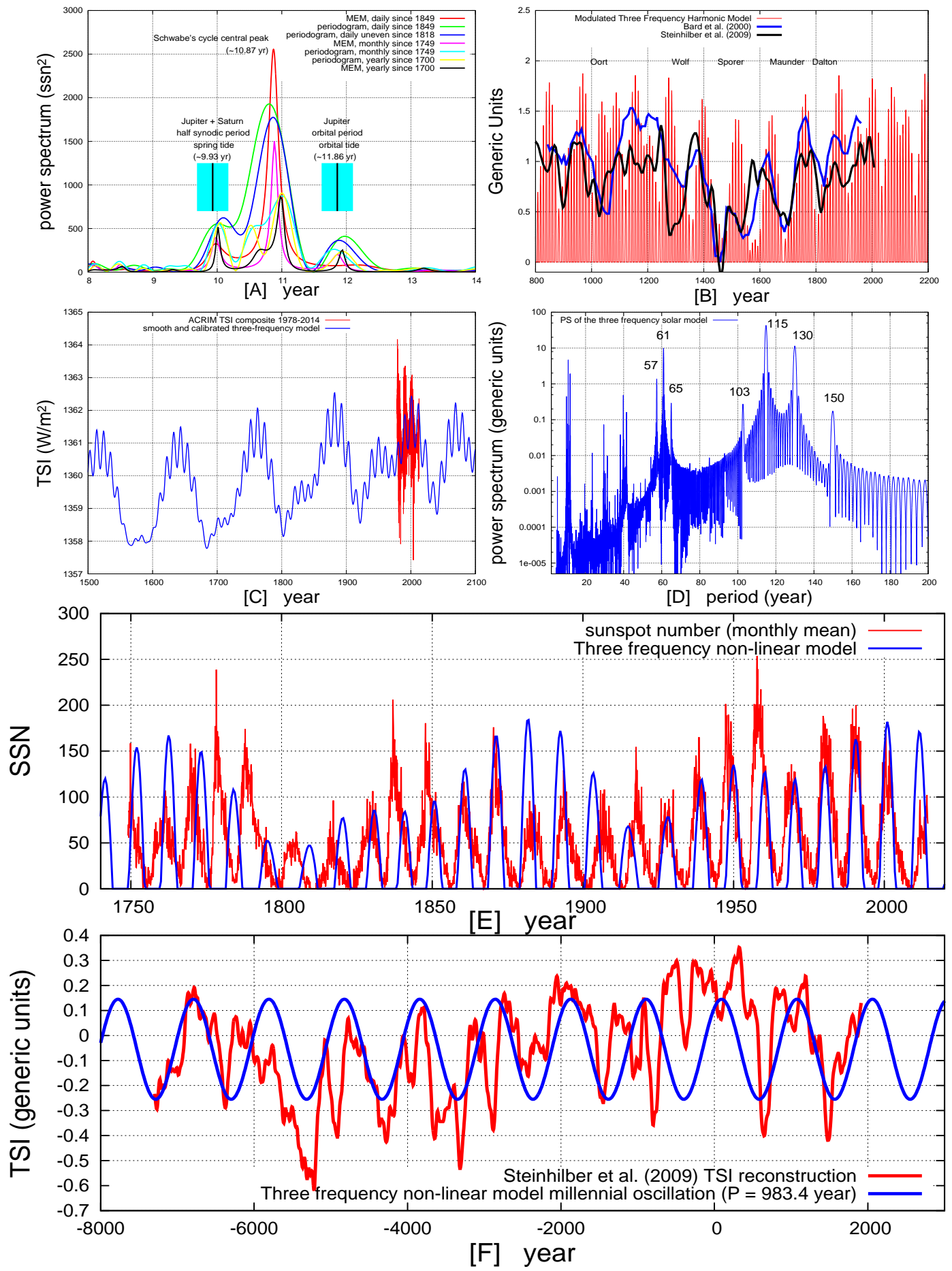

Fig. 2 [A] Power spectra of sunspot number records and the three-frequency Schwabe solar cycle (from: Scafetta, 2012c, 2014). When the sunspot record since 1700 is used the statistical uncertenty of the spectral peaks is $\nabla P \approx \pm 0.2$ year using Eq. 3 [B] Comparison of the three-frequency non-linear solar model (Scafetta, 2012c) and nucleotide solar proxy models (Bard et al., 2000; Steinhilber et al., 2012). [C] An attempted smooth of the three-frequency solar model calibrated on the ACRIM total solar irradiance satellite record (Scafetta and Willson, 2014). [D] Periodogram of the three-frequency solar model and main secular frequencies. Note the four spectral peaks at 103, 115, 130 and 150 years. [E] Comparison of the three-frequency non-linear solar model (blue) and the sunspot number record since 1750 (red). [F] Comparison of the millennial oscillation predicted by the three-frequency non-linear solar model (blue) versus the TSI proxy model by Steinhilber et al. (2012) (red).

Scafetta (2012c)'s three-frequency non-linear solar model was proposed and must be interpreted as a first approximation or schematization of solar output variability. Other harmonics generated by the other planets are surely present 
and may add some modulation, but they were ignored because we would like to interpret the periods at 103, 115, 130 and 150 years. The three-frequency solar model produces at least four main theoretical beat frequencies including those at about 61 years (beat between 9.93 and 11.86 year periods), 114.8 years (beat between 9.93 and 10.87 year period), 130.2 years (beat between 10.87 and 11.86 year period) and about 983 years (combined beat among the three harmonics) (Scafetta, 2012c). Similar harmonics are among those typically observed in long solar records (cf. Ogurtsov et al., 2002).

Scafetta (2012c)'s three-frequency non-linear solar model curve is shown in Figure 2B against two nucleotide $\left({ }^{10} \mathrm{Be}\right.$ and ${ }^{14} C$ ) reconstructions of solar activity (Bard et al., 2000; Steinhilber et al., 2012). The schematic signal can be further smoothed and rescaled to actual solar activity records, e.g. the ACRIM total solar irradiance record (Scafetta and Willson, 2014), to represent an ideal solar activity function generated by the chosen three harmonics: see Figure 2C. Both Figures 2B and 2C show a clear 100-150 year synchronized modulation both in the nucleotide solar proxy models and in the three-frequency solar model. The 100-150 year scale reproduced by the model regulates and is in good phase with the grand solar oscillations known as the Wolf, Spörer, Mauder and Dalton grand solar minima. Scafetta (2012c) also showed that the millennial cycle produced by the three-frequency non-linear solar model is in good phase with the millennial oscillation observed in typical solar and climate records throughout the Holocene for about 10,000 years used in Bond et al. (2001). Figure 2F compares the millennial oscillation predicted by the three-frequency non-linear solar model (blue curve) versus the TSI proxy model by Steinhilber et al. (2012) (red curve), and a good phase correlation can be discerned at this time scale throughout the Holocene.

Figure 2D shows the periodogram of the smooth three-frequency solar model record and it shows main spectral peaks at $57,61,65,103,115,130,150$ years, in addition to other minor harmonics and the original three generating frequencies at 9.93, 10.87, 11.86 years. The frequencies highlighted in Figure 2D are independent of the smooth algorithm and are found in the record depicted in Figure 2B. Aliasing artifacts noted by Poluianov and Usoskin (2014) referring to the model proposed by Abreu et al. (2012) are not present in my analysis because the three-frequency model is constructed with three decadal harmonics and the periodogram is calculated with a record sampled every 6 months for 10,000 years.

In conclusion, both Cauquoin et al. (2014) and Steinhilber et al. (2012) ${ }^{10} \mathrm{Be}$ and ${ }^{14} \mathrm{C}$ solar proxy models present 4 common spectral peaks at about 103, 115, 130 and 150 years (confidence level 95\% or larger). Two of these frequencies appear to be predicted by Abreu et al. (2012) planetary model, although with some doubt (Poluianov and Usoskin, 2014). However, all four frequencies are predicted by the three-frequency non-linear solar-planetary model proposed by Scafetta (2012c) that combines the major Jupiter and Saturn's planetary decadal harmonics with the Schwabe 11-year solar cycle and further requires a non-linear processing, whose existence is very reasonable given the fact that both solar and geophysical systems are non-linear.

Abreu et al. (2012)'s model does not reproduce these cycles because the four oscillations at 103, 115, 130 and 150 year emerge from the modulation of the 10.87 year central sunspot cycle, which is likely generated by the solar dynamo, by the two side Jupiter and Saturn planetary tidal harmonics shown in Figure 2A plus the nonlinear solar response to external harmonic forcing. Abreu et al. (2012)'s model does not reproduce the 11-year solar cycle and, therefore, it cannot reproduce the four secular oscillations found in the solar proxy records depicted in Figure 1. 
In conclusion, the analysis contradicts Cauquoin et al. (2014)'s conclusion. I have demonstrated that common evidences do exist for a planetary influence on solar activity 330,000 years ago and during the Holocene once that the appropriate planetary-solar model, that is the three-frequency non-linear solar model (Scafetta, 2012c) d, see also the Appendix), is used. Other harmonics found in Abreu et al. (2012) can be interpreted in alternative ways. For example, the $\sim$ 87-year harmonic seems to be related to Jupiter, Uranus and Neptune (cf: Scafetta and Willson, 2013a), and the $\sim$ 207-year harmonic may be a beat between the $\sim$ 61-year harmonic and $\sim$ 87-year harmonic that, through non-linear processing, produces a $\sim 205$-year cycle.

\section{The decadal and secular scale spectral coherence between the solar system and the Earth's global surface temperature}

Scafetta $(2010,2012 \mathrm{a}, \mathrm{b}, 2013 \mathrm{a}, \mathrm{b})$ found that the climate system since 1850 presents evidences of multiple astronomical harmonics at the periods of about 5.2 year, 5.93 year, 6.62 year, 7.42 year, 9.1 year (main solar-lunar tide cycle), 10.4 year (related to the 9.93-10.87-11.86 year solar cycle harmonics), 13.8 year, $\sim 20$ year, $\sim 30$ year and $\sim 61$ year (cf.: Scafetta, 2010, 2012a d d, 2014; Wang et al., 2012). This property was found by direct comparison between the temperature and astronomical spectra and by taking into account evidences for paleoclimatic temperature oscillations and the basic harmonics known from astronomy. For the benefit of the reader Figure 12 in the Appendix reproduces figure 6B and 9A of Scafetta (2010) showing [A] the $\chi^{2}$ spectral coherence test and [B] the direct comparison between the MEM curve of several climatic records and the astronomical, solar and lunar harmonics (green bars). The spectral coherence between the two systems is quite evident at multiple periods.

Using time frequency analysis based on $L=60$ year moving windows and magnitude squared coherence analysis based on $L=20$ and 30 year moving windows Holm (2014a) questioned my results by claiming that he could find only a frequency "line in the 15-20 year range which varies with time as well as one around 9 years which comes and goes and varies in frequency": see Figure 3. Holm (2014a) interpreted his results as contradicting Scafetta's hypothesis of a possible astronomical origin of the solar/temperature oscillations because Scafetta's astronomical hypothesis would imply multiple time quasi-invariant temperature spectral lines at specific astronomical frequencies.

It is evident, however, that results are not independent of the mathematical methodology adopted for the analysis. Specific physical properties can be well highlighted with an analysis methodology but not equally well with a different one. Thus, the mere fact that Holm could not find Scafetta's results by using a different analysis methodology is not a sufficient argument to dismiss Scafetta's claims.

Below I will clarify several physical and mathematical issues and demonstrate that Holm's conclusion is flawed because his spectral analysis does not have a sufficient spectral resolution and, therefore, it was highly inadequate to properly identify the harmonics of interest and determine the expected spectral coherence patterns found in Scafetta (2010). 

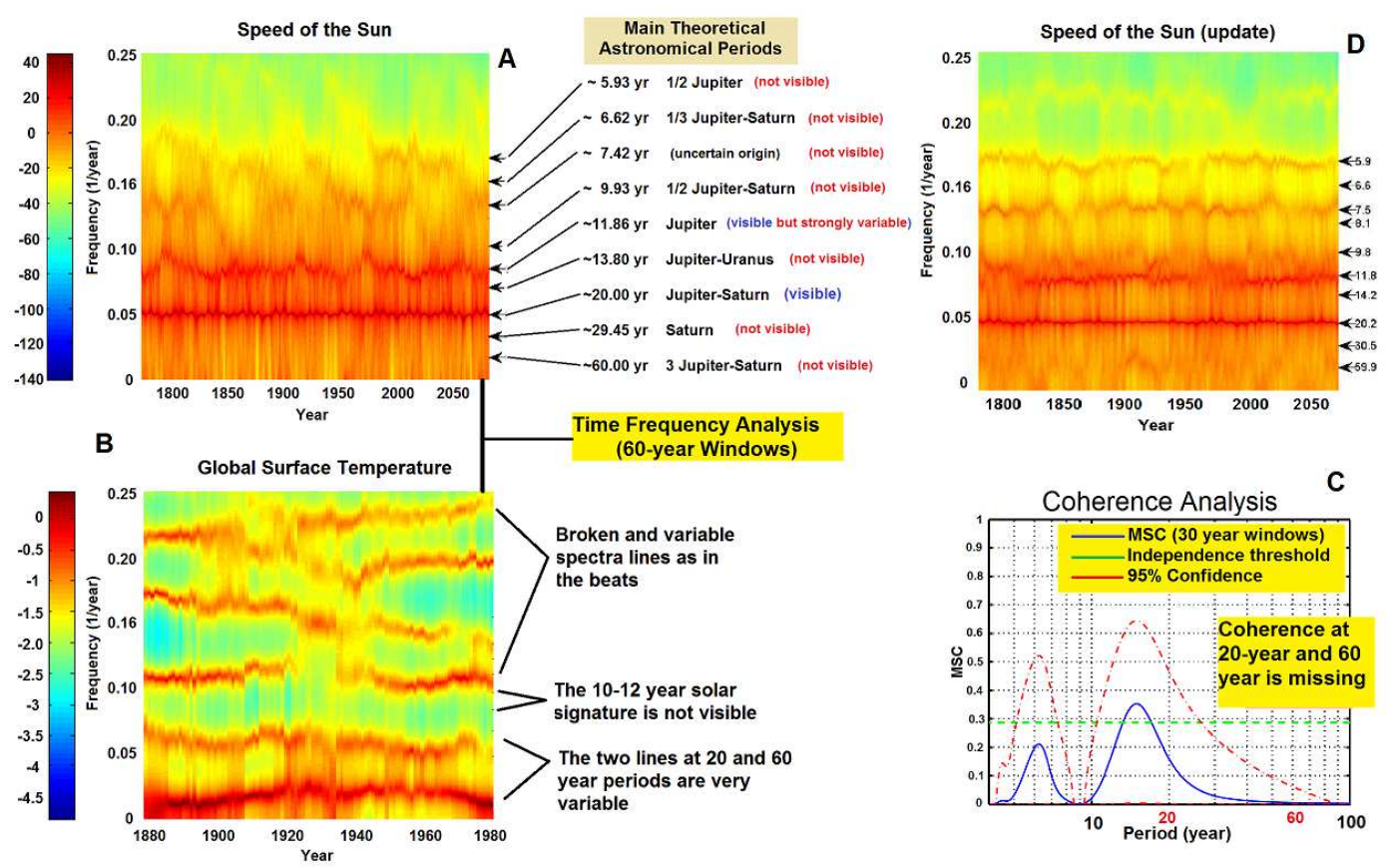

Fig. 3 [A,B,D] Time frequency analysis with Maximum Entropy Method (MEM) power spectrum with an order M=42\% of the window length. [A] Speed of the Sun relative to the barycenter of the solar system (using a windows length $L<60$ years, probably $L=40$ years). [B] Earth's global surface temperature. [C] Magnitude squared coherence using $L=30$ year windows between the two records: peaks are at 6.3 and 14.9 years. The embedded table lists the main (orbital and synodic) harmonics theoretically expected in the astronomical record. Most of them are not visible in the Holm's graph, or their signature is strongly intermittent and variable. Compare with Figures 8 and 10 where the analysis is repeated with $L=110$ years. The figures are adapted from figures 2, 3 and 5 in Holm (2014a). Figure D shows a corrigendum of Figure 3A based on a windows length $L=60$ years ( $\mathrm{Holm}, 2014 \mathrm{~b})$, but still many astronomical harmonics are missing and/or strongly variable.

\subsection{Summary of Holm's analysis}

Figure 3A shows Holm (2014a, figure 3)'s time frequency analysis of the speed of the Sun relative to the barycenter of the solar system. This figure already suggests that Holm's time frequency analysis is inadequate to identify the harmonics of interest. In fact, among the main theoretical astronomical harmonics (reported in Figure 3 as well) only the strong $\sim 20-$ year synodic period between Jupiter and Saturn is clearly visible. Jupiter's 11.86-year orbital period is weakly visible and very unstable: it appears to vary between 10 and 14 years. All other expected astronomical harmonics are not visible or are strongly intermittent and variable.

Holm himself seemed to be partially aware of the problem and in April, 2014 he published on arXiv a corrigendum (Holm, 2014b) with an updated figure 3 herein reported in Figure 3D. This corrigendum was required because Holm (2014a) claimed that his figure 3 (depicted herein in Figure 3A) used a time frequency analysis with window length $L=60$ years, while indeed the graph was based on a shorter window length ( $L=40$ years). His updated figure (depicted herein in Figure 3D) shows the analysis with window length $L=60$ years. Now, the astronomical frequencies at periods of 5.9 and 7.4 years appear, and the 11.86 year Jupiter orbital period becomes more stable. However, many expected astronomical harmonics are still missing, and those visible are strongly variable with the exception of the dominant 20-year Jupiter-Saturn synodic oscillation. Yet, the astronomical harmonics are quite stable in reality.

The missing and/or variable spectral line problem is due to the adoption of a too short window length $L$ in the spectral analysis that yields an inadequate spectral resolution required to solve close harmonics. Indeed, comparing 
Figure 3A (using $L=40$ years) and its updated Figure 3D (using $L=60$ years) clearly suggests that by simply increasing the time frequency window length, new spectral lines appear and others become more stable. Holm's also used a too small MEM order M. He used $M=42 \%$ of the data length, while it would have been better to use the maximum available value, that is $M=50 \%$ of the data length, because of the very short windows he used. The same problem also corrupts Holm's time frequency analysis for the temperature record shown in Figure 3B and his spectral coherence analysis.

3.2 Understanding the first and second law of Kepler, and the data patterns

Some of the shortcomings of Holm's analysis are self-evident from his own figures once basic astronomy is taken into account. The purpose of Scafetta (2010)'s analysis was to identify the astronomical frequencies and test whether they could be found in the climate records. However, in his coherence figures 4 and 5 , which were made using windows with lengths of $L=20$ and 30 years respectively, Holm (2014a) could not find Scafetta (2010, figures 6, 9 and 10 )'s spectral coherence between the Earth's temperature and the astronomical record at the $\sim 20$ and $\sim 60$ year periods, as well as at other frequencies. Compare versus Scafetta (2010) results briefly summarized in Figure 12 in the Appendix.

Missing the coherence at the 20-year period is paradoxical because in his own time frequency analysis Holm could find a quasi 20-year oscillation in both the temperature and astronomical record: see Figure 3. On the contrary, the missing coherence at the 60-year period was due to the fact that while Holm could find a quasi 60-year oscillation in the temperature record (see Figure 3B), both in his original and in his updated figure (see Figures 3A and 3D) he could not find a distinct 60-year oscillation in the astronomical record. In Section 3.9 I will show that this failure is due to the $L=20$ and 30 year windows used in his coherence analysis, which are even shorter than the $L=60$ year window used in his time frequency analysis.

Note that the same $L=60$ year time frequency technique could find the 60-year oscillation in the temperature record but not equally well in the astronomical record because the sensitivity of spectral analysis also depends on the actual spectral power of the harmonics. Small harmonics are harder to highlight if their period is close to the length of the data and other close harmonics have a larger power. In the temperature record the 60 -year oscillation is the dominant cycle as shown in Section 3.6, while in the chosen astronomical record the 60-year oscillation is a secondary cycle and is significantly smaller than the 20-year cycle. Yet, a 60-year cycle is clearly visible in the JPL's HORIZONS ephemeris of the Sun's barycentric speed after some moving average filtering to remove the 20-year oscillation: see Figure 4C (cf. Scafetta, 2010, 2014).

The existence of a 60-year astronomical oscillation cannot be questioned in the barycentric speed of the sun because it derives directly from the 5/2 resonance between 5 Jupiter's revolutions (orbital period $=11.86$ year) and 2 Saturn's revolutions (orbital period $=29.46$ year). The quasi 60-year oscillation emerges in the barycentric movement of the Sun because according the first law of Kepler the orbits of Jupiter and Saturn are elliptical; and according the second law of Kepler their speeds change in function of the planetary coordinates and determine the dynamics of the Sun's barycentric wobbling. 
Jupiter-Saturn conjunctions occur every 19.86 years and two consecutive ones are separated by about $242.8^{\circ}:$ see Figure 13 in the Appendix. Thus, every three consecutive conjunctions, that is a trigon or about 60 years, a Jupiter-Saturn conjunction occurs approximately at the same position of the sky and, therefore, their combined position and velocity repeats not only every $\sim 20$ year but also every $\sim 60$ years. The trigon slightly rotates every $800-1000$ years giving origin to an additional quasi millennial oscillation: see the detailed discussion in Scafetta (2012a), http://en.wikipedia.org/wiki/Great_conjunc The 20, 60 and 800-1000 year Jupiter-Saturn conjunction oscillations were even well-known since antiquity and linked to historical events that today would be linked to climate changes (cf. Ma'sar, 886; Kepler, 1606): for example the 60-year oscillation was chosen as the basis for the traditional Indian and Chinese calendars, where it is known as the Brihaspati (that means Jupiter) cycle, and likely linked to the monsoon 60-year cycles (cf. Agnihotri and Dutta, 2003; Iyengar, 2009; Temple, 1998).

From the above, it is evident that Holm's failure of clearly detecting the 60-year oscillation in the Sun's movement could only mean that Holm's analysis was inadequate to detect accurately the elliptical shape and the dynamics of the orbits of Jupiter and Saturn in the Sun's speed record. Therefore, Holm's methodologies had to fail to find the spectral coherence between astronomical and temperature frequencies because they could not find the frequencies of interest in the first place.

In any case, it is also important to highlight that if the planetary record is substituted with the tidal function, the quasi 60-year oscillation emerges as a dominant beat cycle between the 9.93-year Jupiter-Saturn spring tidal harmonic and the 11.86-year Jupiter orbital tidal harmonic, which non-linear mechanisms transform into an explicit harmonic: see Figure 2 (Scafetta, 2012cd). Therefore, there are several ways to interpret the quasi 60-year oscillation using planetary harmonics.

3.3 The complex planetary synchronization structure of the solar system

To test whether the climate presents a signature of astronomical frequencies the first step is to determine them. Studying their physical and mathematical properties is necessary to select the appropriate analysis methodology to be used for the task.

It is important to understand that Scafetta (2010) used the speed of the Sun relative to the barycenter of the solar system only and exclusively as a proxy for easily determining the main frequencies of the gravitational oscillations of the heliosphere. Using different planetary functions is expected to yield similar frequencies because any function of the planetary orbits present the same set of frequencies by algebraic construction. However, each individual function stresses them differently by showing alternative spectral amplitudes.

On the contrary, to deduce the physical amplitudes all physical mechanisms linking the astronomical to the climate records need to be identified. These also include the internal resonance frequencies of the Sun and of the climate system, and their numerous feedback mechanisms. To develop a full physical model that explains in details both solar dynamics and the Earth' climate using planetary forces is far beyond the purpose of the present paper and of the present scientific knowledge. 

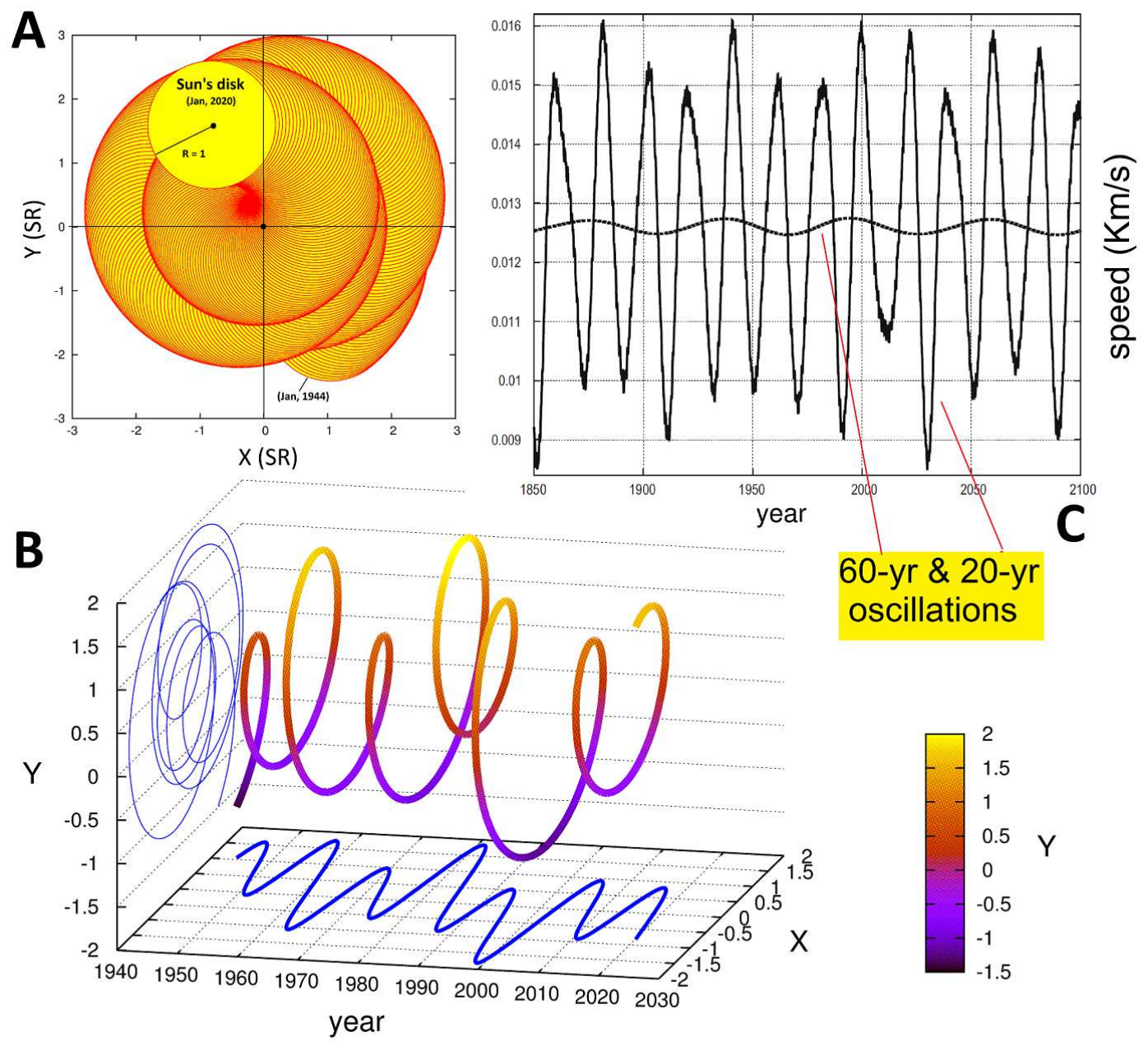

$60-y r \& 20-y r$ oscillations

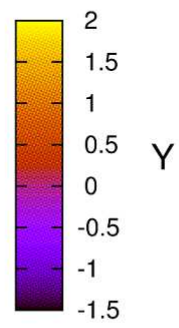

Fig. 4 The wobbling of the Sun relative to the center of mass of the solar system. (A) Monthly scale movement of the Sun from 1944 to 2020 as seen from the $\mathrm{z}$ axis perpendicular to the ecliptic. The Sun is represented by a moving yellow disk with a red circumference. (B) The trajectory of the center of the Sun from 1944 to 2020. (C) The speed of the Sun from 1850 to 2100. Note the evident 20-year and 60-year oscillations: the latter is highlighted by the smooth dash curve. The Sun's coordinates are estimated using the Jet Propulsion Lab's (JPL) HORIZONS Ephemeris system. The coordinates are expressed in solar radius (SR) units. The figures are adapted from Scafetta (2010, 2014).

The frequencies of interest can be theoretically deduced from astronomical considerations alone by taking into account the orbital periods of the planets, their combinations and various sub-harmonics. However, numerous frequencies are expected, and it is simpler to calculate them by analyzing the power spectrum of a planetary proxy record such as those describing the Sun's barycentric movement.

Scafetta (2014) used the JPL's HORIZONS Ephemeris system to calculate the wobbling and the speed of the Sun from 12 December 8002 BC to 24 April 9001 AD (100-day steps): see Figure 4. Perhaps, the speed of the wobbling Sun can have an electromagnetic interaction meaning. On the contrary, for gravitational interactions the tidal function should be used and an example was discussed in the previous section that stresses differently some harmonics, see Figure 2 (Scafetta, 2012a) d d Scafetta and Willson, 2013b a). However, here the issue is to determine the basic frequencies of the solar system. 
Power spectrum of the Sun's speed was evaluated using the Multi Taper Method (MTM) (Ghil et al.,,2002): see Figure 5. The sub-annual spectrum of this record is shown and studied in Scafetta and Willson (2013b), where it was found coherent with several total solar irradiance high frequency harmonics.

Main spectral peaks at about 5.93, 6.63, 7.42, 9.93, 11.86, 13.8, 20, 60.9 years are clearly observed: see the table depicted in Figure 3 for their physical attribution. Note that some spectral peaks are common with the independent threefrequency solar model based on tidal cycles and the sunspot record (Figure 2D) such as the triplet at 57,60.9 and 65 year period that emerges from an asymmetry of the 60-year trigon cycle.

A major physical result of the analysis is that, as shown in Figure 5, from the annual to the secular scale, the natural frequencies of the solar system (shown by the peaks in the red MTM curve) are approximately reproduced by the following simple empirical harmonic formula (Jakubcová and Pick, 1986; Scafetta, 2014):

$$
f_{i}=\frac{1}{P_{i}} \approx \frac{i}{178.4} \quad y r^{-1}, \quad i=1,2,3, \ldots
$$

Eq. 1 suggests that the solar system is almost locked in a synchronization pattern made of a specific harmonic series. The finding can be considered compatible with Kepler's vision of a cosmographic mystery and of the harmonices mundi (the harmony of the world), known since Pythagoras of Samos as musica universalis (universal music or music of the spheres). Because the entire solar system appears to be subtly self-synchronized in a harmonic sequence, the frequencies of its coupled gravitational structure could be expected to influence also solar dynamics and the Earth's climate.

In fact, as discovered by Huygens in the 17th century, in absence of any significant noise or friction (in the space there is none) coupled oscillators (e.g. the Sun, the planets and the moons of the solar system) can mutually synchronize also if the strength of the physical interactions is quite weak (Pikovsky et al., 2001). Scafetta (2014) provided a general introduction on the complex planetary synchronization structure of the solar system. There a reader can find a review of the relevant research. This includes topics such as: Copernicus and Kepler's vision of a cosmographic mystery revealing a "marvelous proportion of the celestial spheres" referring to the "number, magnitude, and periodic motions of the heavens" (Copernicus, 1543; Kepler, 1596); the planetary rhythm of the Titius-Bode rule (Titius, 1766); the asteroid belt mirror symmetry rule (Geddes and King-Hele, 1983); the matrix of planetary resonances (Molchanov, 1968, 1969a) b); the 2/3 synchronization of Venus axis rotation period with the Earth's orbital period so that at every inferior Venus-Earth conjunction the same side of Venus faces Earth (Goldreich and Peale, 1966); the existence of a mathematical relation linking planetary orbital parameters (Tattersall, 2013); the existence of several other celestial commensurabilities such as the quasi synchronization between the 27.3-year lunar orbital period with the 27.3-year Carrington solar rotation cycle as seen from Earth; and many others.

Figure 5 also shows in cyan the major theoretical lunar tidal harmonics deduced from the 18.6-year lunar nodal cycle, the 18.03-year Saros cycle, the 8.85 apside rotation cycle and their second, third and forth harmonics. The yellow areas represent the Schwabe 11-year solar cycle band, the Hale 22-year solar magnetic cycle band and the 100-150 year solar band discussed in Figure 2. 


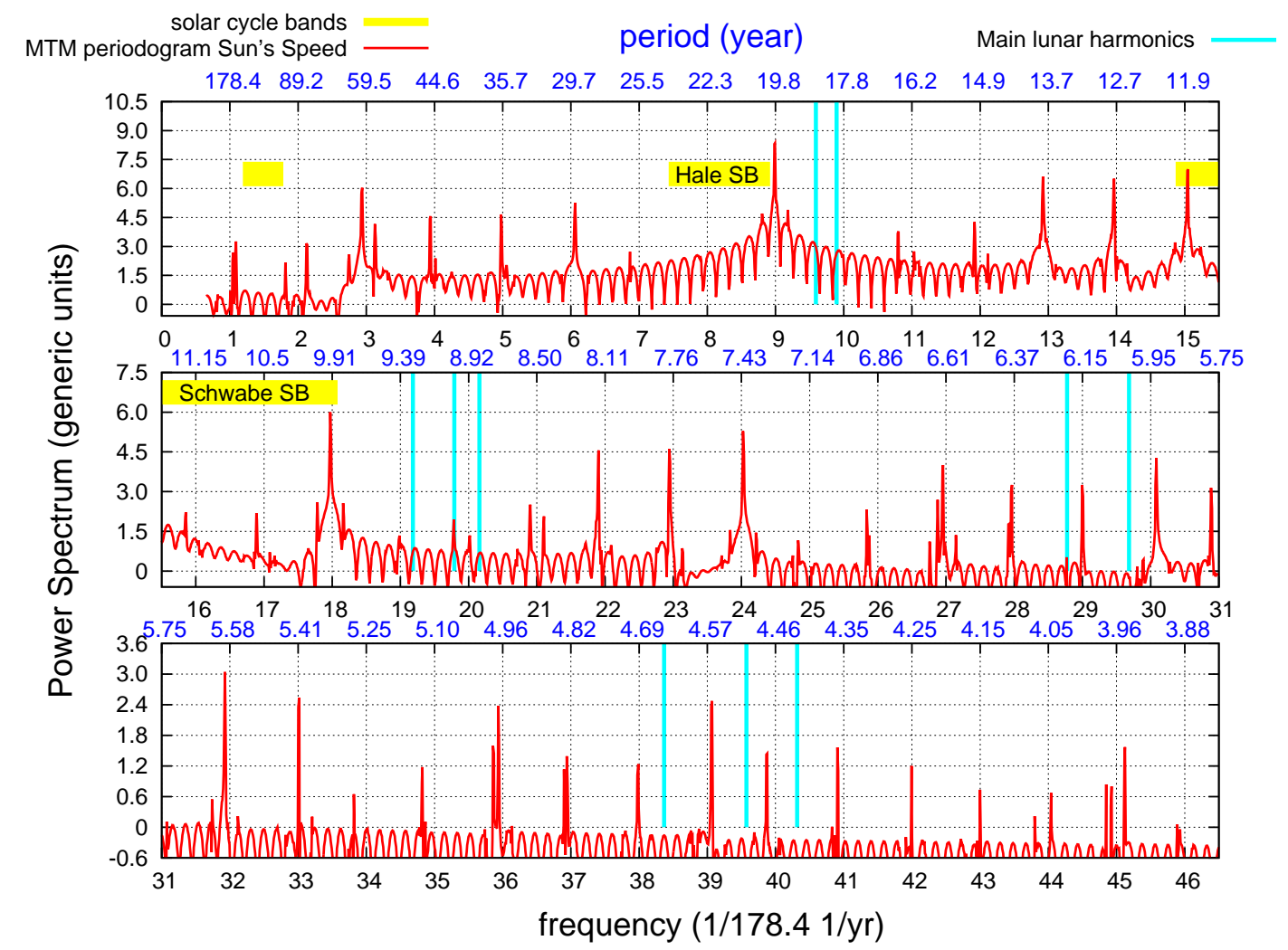

Fig. 5 MTM periodogram (red) of the speed of the Sun relative to the center of mass of the solar system from Dec 128002 B.C. to 24 Apr 9001 A.D. All spectral peaks are highly significant because the astronomical record has likely of 6-7 digit precision and the peaks are sharp and can be recognized from astronomical consideration alone because correspond to the orbital periods, the synodic periods and their harmonics. The cyan lines correspond to theoretical lunar tidal harmonics that can influence the climate: the 18.6, 18.03, 8.85 year cycles and their harmonics. The yellow areas correspond to the Schwabe 11-year solar cycle band, to the Hale 22-year solar cycle band and the 100-150 year solar band discussed in Figure 2.

The frequencies shown in Figure 5 represent the theoretical astronomical harmonics that could be reasonable expected to influence the climate.

3.4 Understanding the spectral resolution of the time frequency analysis

The astronomical sequence analyzed in Figure 5 is about 17,000 years long and its spectral peaks are sharp and many of them can be easily recognized from simple astronomical considerations alone. However, short sequences theoretically made of the same harmonics are difficult to analyze because close harmonics generate complex low frequency beats that need to be solved by the spectral analysis to separate them. In fact, the closer the frequencies, the longer the segment must be to conclude that more than one frequency is present within a specific spectral band. If the segment is too short, two close waveforms will look like a single sine wave and the spectral technique fails to separate them. In brief, as well known, the length of the signal limits the frequency resolution of the spectral analysis. Let us clarify the issue using mathematics.

If $T$ is the acquisition time of a sequence, $N$ is the number of acquired samples and $f_{s}$ is the sampling frequency, the frequency resolution of its Fourier analysis is defined as (FFT): 


$$
\nabla f=\frac{1}{T}=\frac{f_{s}}{N}
$$

A frequency $f$ associated with a spectral peak has an uncertainty of $\pm 1 / 2 \nabla f$ and the correspondent period $P$ is:

$$
P=\frac{1}{f \mp 1 / 2 \nabla f}=\frac{1}{f} \pm \frac{\nabla f}{2 f^{2}}=\frac{1}{f} \pm \frac{P^{2}}{2 T} .
$$

To well separate two frequencies $f_{1}=1 / P_{1}$ and $f_{2}=1 / P_{2}$, the following condition must be fulfilled

$$
f_{12}=\left|f_{1}-f_{2}\right| \gtrsim \nabla f,
$$

where, as well known, $f_{12}=\left|f_{1}-f_{2}\right|$ is the beat frequency between the two frequencies. Consequently to well separate two close frequencies using Fourier based spectrum analysis the acquisition time $T$ must be about or larger than the beat period, $P_{12}=1 / f_{12}$, between the two contiguous harmonics, that is:

$$
T=\frac{1}{\nabla f} \gtrsim \frac{1}{\left|f_{1}-f_{2}\right|}=P_{12}
$$

The Maximum Entropy Method (MEM) produces sharper spectral peaks with smaller error bars, but MEM can also produce spurious spectral peaks. For this reason it is conventional to validated the MEM spectral peaks using Fourier based periodograms (Press et al., 1997), and then use MEM to sharp the results, as I have often done (e.g.: Scafetta, 2010, $2012 b, 2013 b)$. So, for safety, the condition of Eq. 5 must be used also when MEM is applied.

Time-frequency analysis divides a sequence in moving window segments of length $L$ and evaluates the spectra of these segments to study how the spectral energy evolves in time. Eq. 5 implies that, when time-frequency analysis is used to determine whether a sequence contains a specific set of time-invariant spectral lines, it is necessary to chose its moving-window length $L$ to be about or longer than the theoretical beat periods among the proposed harmonic constituents of the signal. If $L \ll P_{\text {beat }}$ time-frequency analysis only highlights the interference dynamics of the beats among the constituent harmonics producing variable patterns such as those visible in Figure 3.

Let us explain this result with a simple exercise that also serves to illustrate the inadequacy of Holm's 60-year and shorter window methodologies. Figure 6A shows an artificial sequence of 200 years made of two harmonics with periods of 10 and 11 years plus random noise. Figure 6B shows its MEM time-frequency analysis using 60-year windows as chosen in Holm (2014a). It is evident that with $L=60$ years the technique fails to emphasize the presence of the two constituent time-invariant harmonics by producing a variable beating pattern. In fact, the frequency resolution associated to a 60 -year window is $\nabla f_{60}=1 / 60=0.0167$. This value is significantly larger than the minimum spectral resolution required to separate the two harmonics, which is $\nabla f_{10,11}=1 / 10-1 / 11=1 / 110=0.0091$. Figure $6 \mathrm{C}$ shows the time-frequency analysis of the signal using $L=110$ year windows, which corresponds to the beat period. Now the time-frequency analysis clearly reveals the two expected time-invariant spectral constituent lines at the periods of 10 and 11 years. 


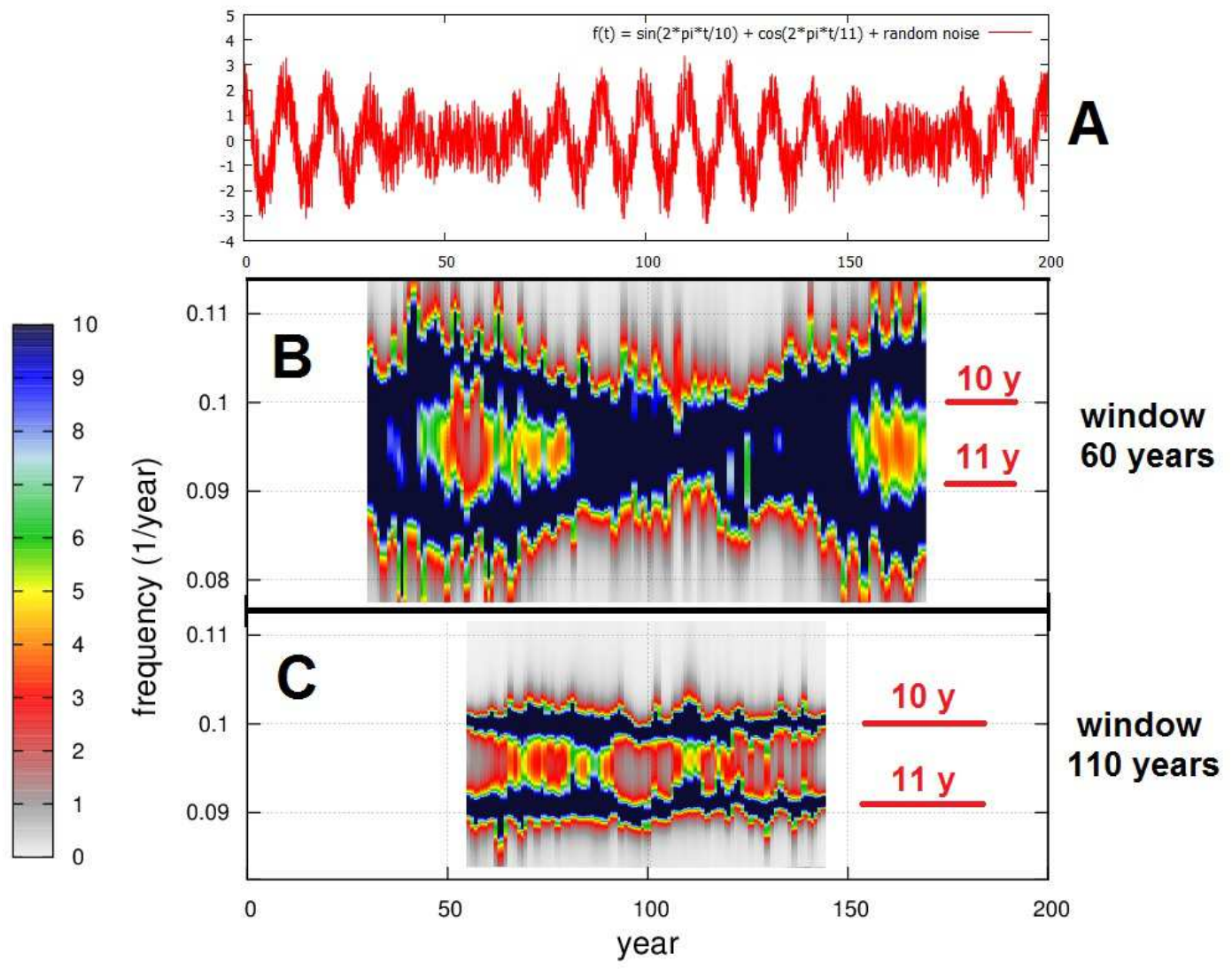

Fig. 6 [A] Artificial sequence made of two harmonics with periods of 10 and 11 years, plus random noise: the record shows a clear periodic beat with a 110-year period. [B] Time-frequency analysis of the signal using MEM and $L=60$ year moving windows. A variable pattern is observed. [C] Time-frequency analysis of the signal using MEM and $L=110$ year moving windows, which is the theoretical beat period. The two constituent harmonics are highlighted by the analysis. The MEM order is $\mathrm{M}=50 \%$ of the available points $(M=N / 2=360$ and $M=660$, respectively). The panel colors are in generic units from a minimum power indicated as " 0 " to a maximum indicated as " 10 ".

3.5 The optimal spectral window length

In choosing the most appropriate window for his time frequency analysis Holm (2014a) simply argued: "The length of the data window must be chosen and the longer the window, the better the frequency resolution. On the other hand, the shorter the window, the better the ability to track time variations in the data. For the examples shown here, a window length of 60 years was found to be a reasonable compromise." However, it appears that Holm's "reasonable compromise" was based only on a qualitative personal impression. In fact, it was not based on any mathematical or physical property of the data.

It is true that using windows as short as possible increases the number of independent spectra that can be compared. However, Holm ignored that, to be useful, the frequency resolution of the methodology must be appropriate to separate the harmonics of interest. Thus, the window length could be chosen to be small but not smaller than the largest beat period among the contiguous frequencies that one is interested to identify.

According Eq.11and Figure 5 the theoretical expected astronomical harmonics in the frequency range of interest are approximately sub-harmonics of a 178.4-year oscillation (cf. Iakubcová and Pick, 1986; Scafetta, 2014). This means that the minimal spectral resolution $\nabla f$ required to identify them in both the astronomical and temperature record is 


$$
\nabla f=\frac{1}{L} \lesssim\left|f_{i+1}-f_{i}\right|=\frac{i+1}{178.4}-\frac{i}{178.4}=\frac{1}{178.4}=0.0056 \quad y r^{-1}
$$

Eq. 6 indicates that the spectral window length should be chosen to be $L \gtrsim 178.4$ years. This length is not only far larger than Holm's 20-to-60 year spectral windows, but it is also larger than the available 164-year temperature record. Thus, using a single window equal to the length of the entire temperature record, as done in Scafetta (2010) and below in Table 1, is right now the most appropriate way to study the spectral coherence between astronomical and climatic harmonics. Because 164 years is just slightly shorter than 174 years, MEM could still be reliable to get all main expected harmonics with sufficient sharpness using a single 164-year window under the condition of using the maximum MEM order (M=50\% of the available data), as done in Scafetta (2010).

3.6 Estimation of the expected main beat periods in a 164-year record

To highlight at least the major visible harmonics using time frequency analysis, one needs to study the major beat periods among those clearly visible in a 164-year short record.

Holm (2014a) used the HadCRUT3 global surface temperature record (Brohan, 2006). Herein I re-analyze this record from Jan/1850 to Dec/2013. It is made of 1968 monthly data or 164 annual data. Figure 7A shows the annual temperature record from 1850 to 2013 ( $N=164$ years) with its error bars. Figure 7B shows its power spectrum functions calculated with MEM (using $M=N / 2=82$ ) and with MTM (Ghil et al., 2002).

I used both techniques because, as Press et al. (1997, pp. 574) wrote, "Some experts recommend the use of this algorithm in conjunction with more conservative methods, like periodogram, to help choose the correct model order, and to avoid getting too fooled by spurious spectral features." Essentially, first the periodogram is used to find the spectral peaks with their statistical confidence, then MEM is used to sharp the results. MEM spectral peaks that are not confirmed by the periodogram should be rejected, and vice versa.

Figure 7B does not show any MEM spurious peaks because each peak is confirmed by a correspondent peak in the MTM periodogram. Thus, it is safe to use the maximum MEM order, $M=N / 2$, that adds sharpness to well solve the low frequency range of the record that contains both the long harmonics (e.g. the 20-year and 60-year cycle) and the beat harmonics of the fast oscillations (cf. Scafetta, 2012b, , supplement file).

As Figure 7B shows, most temperature spectral peaks for 4-year and larger periods have a 99\% statistical confidence relative to the physical noise (gray area in Figure 7A) (Ghil et al., 2002). The temperature error has an average standard deviation of $\sigma \approx 0.06^{\circ} \mathrm{C}$. The $95 \%$ and $99 \%$ spectral confidence levels were deduced from computer generated random Gaussian noise with $\sigma \approx 0.06$, and using the SSA-MTM tool kit for spectral analysis (Ghil et al., 2002); they are approximately given by the equation $8.5 \sigma^{2} / \pi(95 \%)$ and $14.6 \sigma^{2} / \pi(99 \%)$, respectively, where $1 / \pi$ is the MTM spectral median for normal Gaussian noise. The MTM spectral peaks have a statistical error given by Eq. 3 For example, for the 60-year cycle the error is \pm 11 years. The MEM and MTM specral peaks are consistent with each other within the MTM spectral errors. 

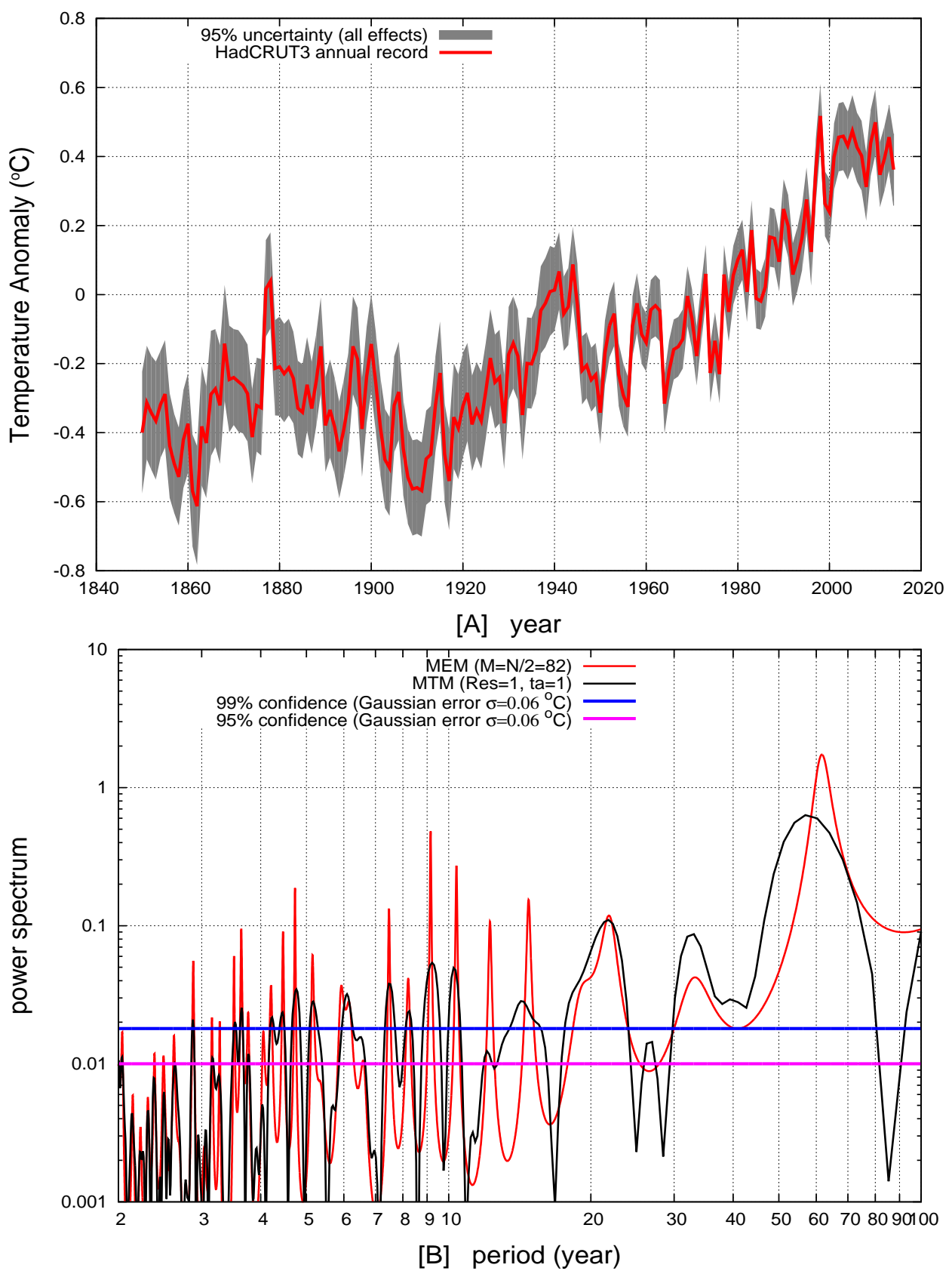

Fig. 7 [A] Annually solved HadCRUT3 record (Brohan, 2006) from 1850 to 2013 (N=164 years). [B] Power spectrum functions calculated with the MEM method (using $\mathrm{M}=\mathrm{N} / 2=82$ ) and the MTM periodogram (Ghil et al., 2002, SSA-MTM Toolkit). The MTM power spectrum statistical confidence levels were deduced using computer generated Gaussian random sequences simulating the combined effects of all known physical temperature uncertainties (annual average has $\sigma \approx 0.06^{\circ} \mathrm{C}$ ). The temperature uncertainties vary in time and are shown in A by the gray area at the $2 \sigma$ level (95\% confidence). (http:/ /www.metoffice.gov.uk/hadobs/hadcrut3/diagnostics/time-series.html)

Table 1 reports: (1) all main spectral peaks found in the temperature record in the range from 4 to 100 year period; (2) the main astronomical expected periods from Figures 3 and 5; (3) $\chi^{2}$ index between the temperature and astronomical periods (values of $\chi^{2} \lesssim 1$ indicate spectral coherence); (4) the theoretical beat periods among the contiguous astronomical frequencies. The $\chi^{2}$ index values between the astronomical and temperature main frequencies reported in Table 1 reveals that these frequencies are coherent to each other within the spectral resolution of the analysis. 


\begin{tabular}{|c|c|c|c|c|}
\hline $\begin{array}{c}\text { Temperature } \\
P_{\text {tem }}(\text { year })\end{array}$ & $\begin{array}{c}\text { Sun's Speed } \\
P_{\text {sun }}(\text { year })\end{array}$ & $\begin{array}{c}\chi^{2} \\
\frac{\left(P_{\text {tem }}-P_{\text {sun }}\right)^{2}}{\left(\nabla P_{\text {tem }}\right)^{2}}\end{array}$ & $\begin{array}{c}\text { close periods } \\
\text { year }: \text { year }\end{array}$ & beat period (y) \\
\hline \hline $5.2 \pm 0.08$ & 5.12 & 1.00 & $5.12: 5.93$ & 37.5 \\
\hline $5.95 \pm 0.11$ & 5.93 & 0.04 & $5.93: 6.63$ & 56.2 \\
\hline $6.54 \pm 0.13$ & 6.63 & 0.48 & $6.63: 7.42$ & 62.3 \\
\hline $7.5 \pm 0.17$ & 7.42 & 0.22 & $7.42: 8.14$ & 83.9 \\
\hline $8.25 \pm 0.21$ & 8.14 & 0.27 & $8.14: 8.85$ & 102 \\
\hline $9.1 \pm 0.25$ & $8.85-9.30^{*}$ & 0.00 & $9.1: 9.93$ & 109 \\
\hline $10.4 \pm 0.33$ & $9.93-11.86^{* *}$ & 0.18 & $11.86: 13.8$ & 84.4 \\
\hline $14.5 \pm 0.7$ & 13.8 & 1 & $13.8: 19.86$ & 45.2 \\
\cline { 2 - 5 } & 15.0 & 0.51 & $15.0: 19.86$ & 61.3 \\
\hline $20.7 \pm 1.2$ & 19.86 & 0.49 & $19.86: 29.45$ & 61 \\
\hline $32 \pm 3.3$ & 29.45 & 0.60 & $29.45: 60.9$ & 57 \\
\hline $61 \pm 11$ & 60.9 & & & \\
\hline
\end{tabular}

Table 1 (1) Spectral peaks of the HadCRUT3 global surface temperature monthly record. The 61-year period has been optimized by comparison with the periodogram and direct filtering (Scafetta, 2010, 2013b). (2) Main spectral peaks of the Sun's speed: from Figure 5. *Range of the solilunar tidal periods which are not found in the Sun's speed. ${ }^{* *}$ Range of the harmonic constituents that make the sunspot cycle, see Figure 2A (Scafetta, 2012C). (3) $\chi^{2}$ index between the temperature and astronomical periods: values $\chi^{2} \lesssim 1$ indicate spectral coherence. (4) Main contiguous astronomical beating frequencies expected to regulate temperature oscillations. (5) Average beat periods. The astronomical spectral resolution is $\nabla f=1 / 17,000=0.00006 \mathrm{yr}^{-1}$, while the temperature spectral resolution is $\nabla f=1 / 164=0.0061 \mathrm{yr}^{-1}$ and it is used for estimating the error of the reported temperature periods using Eq. 3

Table 1 reports that for most frequencies the astronomical-temperature coherence is quite good, $\chi^{2}<1$, while for others is still good but $\chi^{2} \approx 1$. This may be explained by unresolved beats. For example, the temperature shows a spectral peak around 14.5-year period that could be compared with the closest dominant 13.8-year Jupiter-Uranus period. However, Figure 5 reveals that in addition to the 13.8-year harmonic, the astronomical record also contains a 15-year harmonic (=half orbital period of Saturn). So, it is possible that the 14.5-year temperature harmonic is a compromise between the two close astronomical harmonics that the spectral analysis could not well separate. In fact, the beat frequency between the two harmonics is $f_{12}=\left|f_{13.8}-f_{15}\right|=|1 / 13.8-1 / 15|=1 / 172.5=0.0058 \mathrm{yr}^{-1}$, which is shorter than the the spectral resolution available for the 164-year temperature signal, $\nabla f=1 / 164=0.0061$ yr ${ }^{-1}$, Eq. 4. Some spectral disruption in the temperature record may also derive from the smooth anthropogenic and volcano signatures that this record contains (e.g.: Scafetta, 2013b, and many others), but this topic is not addressed here further.

The minimal windows length to be used in a time-frequency analysis should at least be larger than the beat periods listed in Table 1. Scafetta $(2010,2012 \mathrm{a}, \mathrm{b}, \mathrm{c}, 2013 \mathrm{a}, \mathrm{b})$ ) showed that the quasi 9.1-year frequency found in the temperature record may be bounded by the 8.85 years (the lunar apsidal precession) and the 9.3 years (sub-harmonic of the 18.6-year lunar nodal cycle) (cf. Wang et al., 2012) while the 10.4-year frequency is a variable cycle related to the Schwabe solar cycle that derives from a combination of the 9.93-year Jupiter-Saturn spring tidal cycle and the 10.87-year central sunspot number spectral period: the average between the two harmonics is 10.4 years (cf.: Scafetta, 2010, 2012c, 2014). Because the 9.93-year cycle is a harmonic constituent of the 10.4-year cycle, it bounds it. The beat period between the 9.1-year and the 9.93-year harmonics is about 108.9 years and it is larger than the other beat periods listed in Table 1 . Therefore, to highlight the astronomical-temperature coherence at least for the harmonics most visible in a 164-year long sequence, a reasonable minimal window length should be $L \approx 110$ years, not the $L \approx 60$ years used by Holm. 
3.7 Time frequency analysis using 110-year windows

Figure 8 compares the time-frequency analysis of the speed of the Sun relative to the center of mass of the solar system (panel A) and of the HadCRUT3 monthly temperature record (panel B) using $L=110$ year window and $M=N / 2=660$ (50\% of the number of available monthly samples). The figure demonstrates the presence in the temperature panel of multiple time-invariant spectral lines at the major expected astronomical harmonics. The main astronomical periods of interest here are at about 5.2 year, 5.93 year (Jupiter $2 \mathrm{f}$ orbital harmonic), 6.62 year (Jupiter/Saturn 3f synodic harmonic), 7.42 year, 9.1 year (main solar-lunar tide cycle), 10.4 year (related to the 9.93-10.87-11.86 year solar/Jupiter/Saturn harmonics), 13.8 year (Jupiter/Uranus synodic), 19.86 year (Jupiter/Saturn synodic), 29.95 year (Saturn orbital, which is quite weak) and $\sim 60$ year (Jupiter/Saturn triple conjunction and beat tide) (cf.: Scafetta, 2010, 2012a)d,d, 2014; Wang et al., 2012). Among the astronomical harmonics the temperature shows sufficiently stable oscillations at about $7.44,9.1,13.8,20$ and 60 years.

In the 5.93-6.63 range the temperature shows two variable lines that are, however, well bounded by the two major expected theoretical astronomical oscillations. Note that the spectrogram of the entire temperature record (Figure 7B) shows that these two minor harmonics are relatively weak and quite diffused. Probably the used spectral methodology based on 110-year windows was not sufficiently robust to separate well these spectral lines. In fact, according the full spectrogram of the Sun's speed (Figure 5A) the 5.93-6.63 year period range is occupied by 4 astronomical harmonics at 5.93, 6.15, 6.38 and 6.62 year. The $3 f$ harmonic of the 18.6 lunar nodal cycle $(18.6 / 3=6.2$ years $)$ and of the Saros cycle $(18.03 / 3=6.01$ year) are also expected to be present: see Figure 5 . Thus, the 5.93-6.63 year period range could be modulated by five close astronomical harmonics that are beating and could generate the variable pattern observed in Figure $8 \mathrm{~B}$. Windows far longer than $L=110$ years would be required to solve this frequency rang: $L \gtrsim 170$ years if only the Sun's speed harmonics are considered, which can increase up to $L \gtrsim 762$ years if the lunar $3 f$ nodal harmonic are also present. Therefore, the 5.93-6.63 year range cannot be solved well with the current temperature record.

The 10.4-year temperature spectral line is slightly varying between the 9.93-year and 11.86-year astronomical periods, which bound the solar cycle dynamics as seen in Figure 2A (Scafetta, 2012c). The period referring to the variable line at $~ 10.4$ years is a little bit longer at the beginning of the record (up to about 11.86 years) and becomes shorter later (up to about 9.93 years). The evolution of the $\sim 10.4$-year oscillation fits the interpretation that this oscillation is the temperature signature of the variable 11-year solar oscillation. Indeed, from 1843 to 1913 the solar cycle was on average 11-12 years long while from 1913 to 2000 it was on average 10-11 years long (e.g.: Loehle and Scafetta, 2011; Theill and Lassen, 2000; Scafetta, 2012c, table 1). See also Scafetta (2012c) d) for more details about the relation between the solar cycle and the Jupiter-Saturn cycles at 9.93 and 11.86 years, where it is also shown that the probability distribution of the sunspot cycle length since 1750 presents two peaks with a major peak around 10.3 years and a secondary peak around 11.7 years.

There exists also a Venus-Earth-Jupiter model for the quasi 11-year solar oscillation (Hung, 2007; Scafetta, 2012c). In this case the 11-year solar cycle should be bounded between the 10.38-year and 11.68-year average periods, which correspond to 16 and 19 times Venus-Jupiter synodic cycle $\left(P_{V J}=0.6486\right.$ year), respectively. The varying temperature 


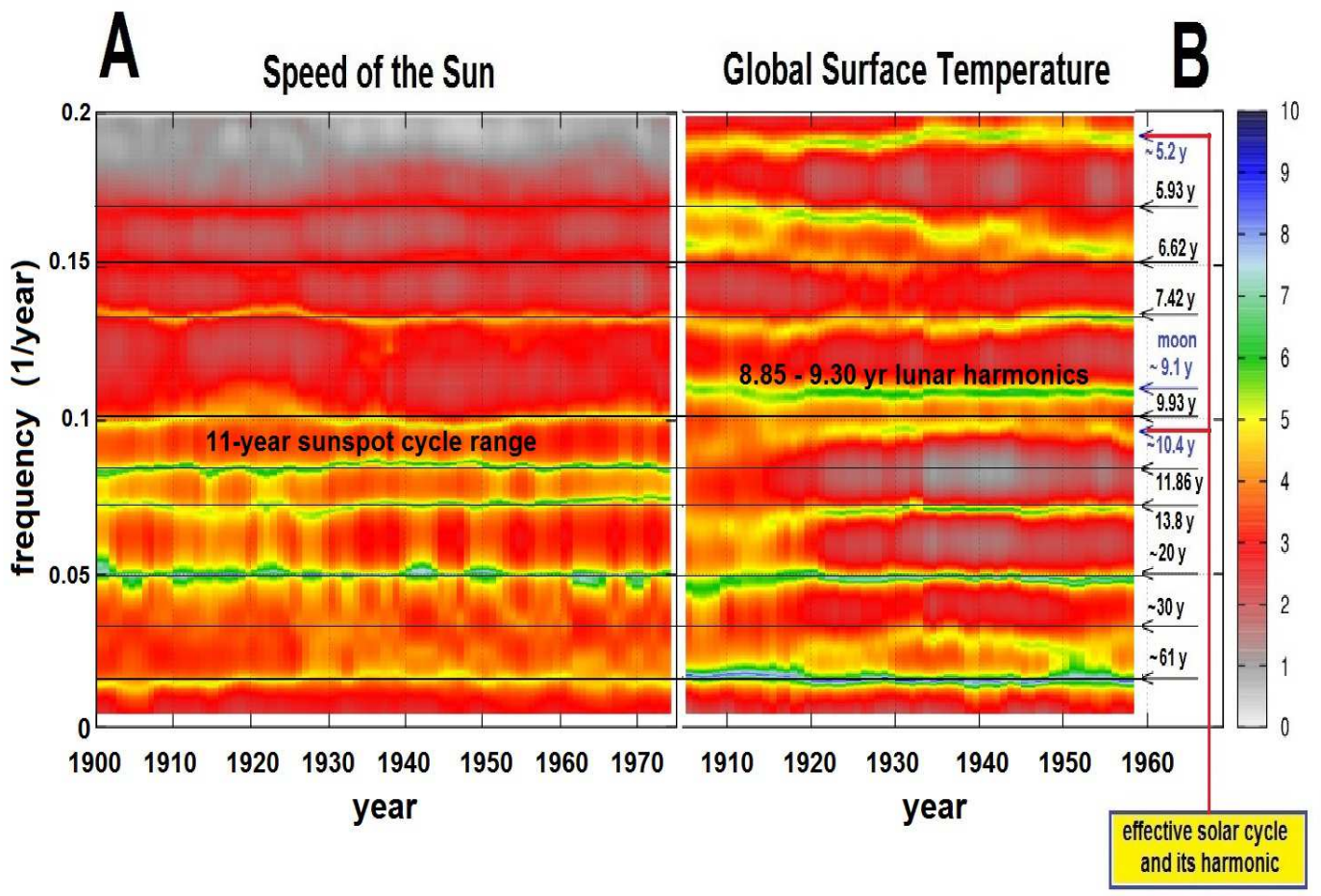

Fig. 8 [A] Time-frequency analysis ( $L=110$ years) of the speed of the Sun relative to the center of mass of the solar system. [B] Time-frequency analysis ( $L=110$ years) of the HadCRUT3 temperature record after a quadratic fit was removed to eliminate the non stationary upward bias (Scafetta, 2010). MEM-based spectrogram M $=660$ (50\% of the number of available monthly samples). The common astronomical timeinvariant spectral lines are highlighted with black straight lines crossing the two panels. The blue arrow indicates the temperature signature of the $\sim 9.1$-year lunar tidal cycle, and the two red arrow the effective temperature signatures of the solar cycle at $\sim 10.4$ years and of its likely harmonic at $\sim 5.2$ years. The panel colors are in generic nonlinear units (to stress small peaks) from a minimum power indicated as " 0 " to a maximum indicated as " $10^{\prime \prime}$. The spectral lines have an uncertainty of $\pm 1 / 2 \nabla f= \pm 1 / 2 / 110= \pm 0.0045 \mathrm{yr}^{-1}$. Note the good spectral coherence at 20 and 60-year periods that Holm (2014a) could not find (cf. Figure 3).

line at 5.2 years could be a harmonic of the 10.4-year line and would correspond to a recurrence made of 8 Venus-Jupiter synodic cycles, which is not well observed in the solar speed record.

3.8 The solar-lunar-astronomical origin of the 8-13 year temperature oscillation band

Let us explicitly clarify what happens in the 8-13 year period band that according to Holm's time frequency analysis should be characterized by a single variable line that breaks down in the interval 1920-1940 (see Figure 3B). This broken and variable pattern gave Holm the impression that Scafetta (2010)'s hypothesis is wrong.

Scafetta (2010, 2012b, 2013b) claimed that the 8-13 year temperature range is mostly regulated by two main astronomical oscillations that, indeed, are quite visible in Figures 7B and 8B: (1) a major solar-lunar tidal oscillation varying between 8.85 years (the period of the recession of the line of the lunar apsides) and 9.3 years (the second harmonics of the lunar nodal cycle), the mean is about 9.1 years ( cf.Wang et al., 2012); (2) a variable 10-12 year Schwabe solar cycle. The temperature spectral peak at 10.4 years is approximately the average between the 9.93-year Jupiter-Saturn spring tidal cycle and the 10.87 central sunspot frequency peak and approximately corresponds to the larger peak of the bimodal solar cycle length distribution Scafetta (2012c, figure 2). 


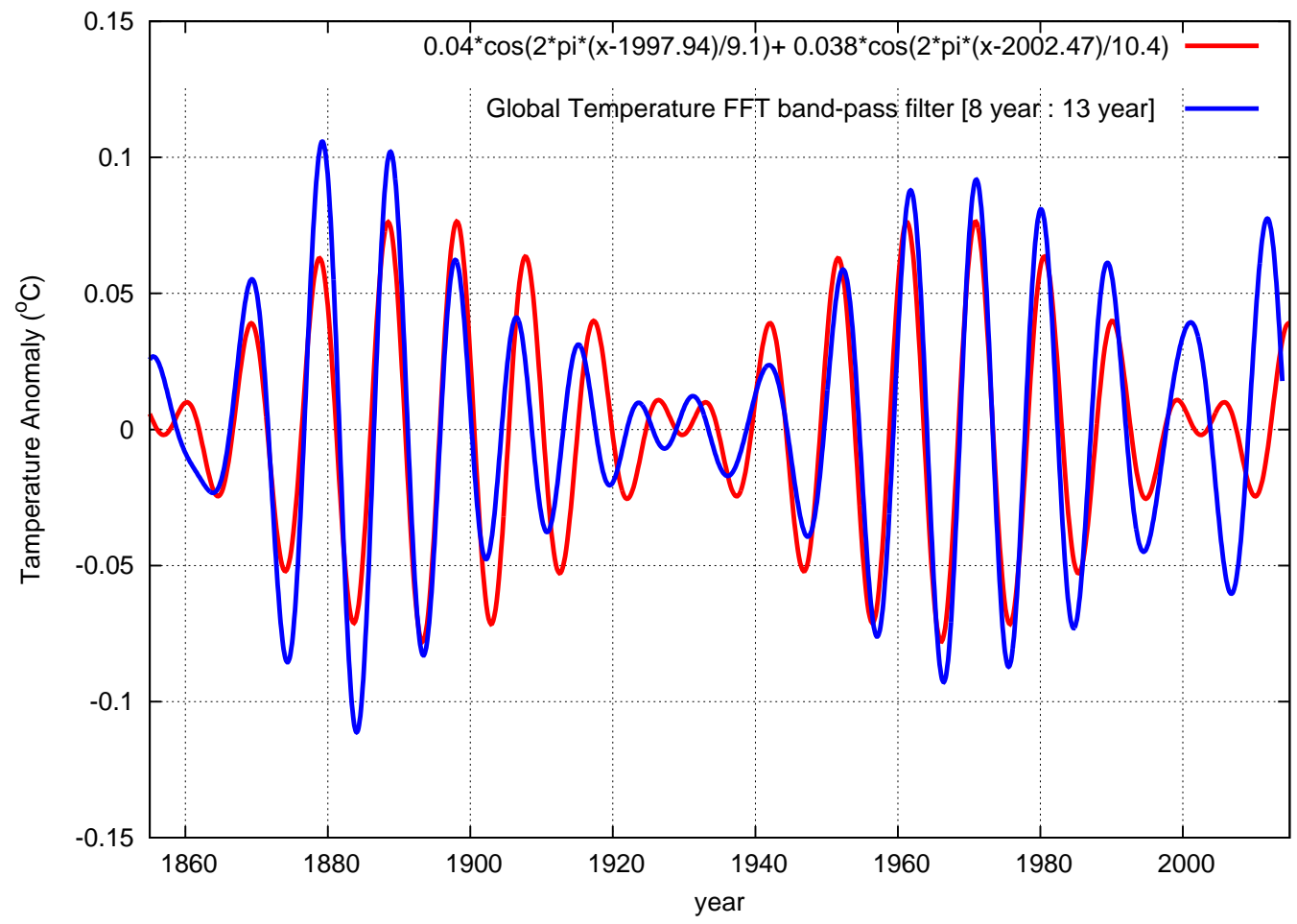

Fig. 9 8-13 year FFT band pass filter 8-13 of the temperature record (blue) and Eq.7(red). The filtering highlights that this frequency range is characterized by a strong beat with a period of about 80 years.

Spectral analysis should advantage the solar cycles in the 10-11 year period range over those in the 11-12 year period range because during the 20th century most solar cycles were short and the Sun is likely more active and the solar cycle amplitude larger during periods characterized by cycles with a short length (cf.: Loehle and Scafetta, 2011; Theill and Lassen, 2000).

Figure 9 shows a FFT band pass filter of the HadCRUT3 record (blue curve) that isolates the global surface temperature variability between 8 and 13 year periods. This record clearly demonstrates the presence of a beat oscillation with a period of about 70-90 years. Thus, at least two major interfering harmonics determine the temperature variability within this range and, according Eq. 5, spectral analysis needs more than 80 years of data to separate them. This evidence again indicates that Holm's chosen window lengths ( $L=20$ to 60 years) were too short and, therefore, inadequate to properly detect the constituent harmonics of the temperature signal.

In particular, Figure 9 clearly suggests that the two harmonics interfere destructively from 1920 to 1940, when the time-frequency analysis implemented by Holm (2014a, figure 2), and reported here in Figure 3B, shows a vanishing pattern.

Because spectral analysis suggests that the two constituent harmonics have average periods of 9.1 and 10.4 years, in first approximation the modulation shown in Figure 9 can be modeled using two harmonics as:

$$
f(t)=0.040 \cos \left(2 \pi \frac{t-1997.94}{9.1}\right)+0.038 \cos \left(2 \pi \frac{t-2002.47}{10.4}\right)
$$

Figure 9 shows that the regression model, $f(t)$, (red curve) well reproduces the filtered temperature curve (blue curve). 
The claim that these two global surface temperature oscillations likely have an astronomical origin is based on two independent observations: (1) the two frequencies match two major astronomical frequency ranges, as explained above; (2) the phases of these two oscillations approximately match the astronomical expectations, as explained below.

In fact, according Eq. 7 the 9.1-year oscillation peaks in 1997.97. During this period the moon was crossing a nodal point at the equinoxes (Scafetta, 2012b, supplement, page 35-36). Moreover, in 1997 the Moon was in its minor declination standstill: a lunar declination standstill occurs every 9.3 years (http://en.wikipedia.org/wiki/Lunar_standstill). Solar and lunar eclipses were occurring in March and September: solar eclipses occurred on 9/Mar/1997, 2/Sep/1997 and 26/Feb/1998; lunar eclipses occurred on 24/Mar/1997, 16/Sep/1997 and 13/Mar/1998 (http://www.timeanddate.com/eclipse/list.l Therefore, the soli-lunar tidal torque was at its maximum because mostly acting on the equator. Probably tidal forces could drive more hot water from the equator toward the poles inducing a maximum in the $\sim 9.1$-year global surface temperature oscillation. A climatic effect of the tidal forces moving the ocean water at different latitudes may be relatively quick.

According Eq. 7 the 10.4-year temperature oscillation peaks in 2002.47 (Scafetta, 2012c). Several solar indexes, such as total solar irradiance, peaked around 2002 (e.g.: Scafetta and Willson, 2013b, 2014) and the Jupiter-Saturn conjunction occurred in 2000.475 (Scafetta, 2012c). Therefore, the maximum of the combination of the two astronomical oscillations can be theoretically expected to have occurred between 2001 and 2002. A 1-year time-lag between the decadal solar/astronomical oscillation and the equivalent temperature oscillation that peaked around 2002.5 is physically plausible because of the thermal heat capacity of the climate system (Wigley, 1988). A more detailed analysis is left to another dedicated study.

3.9 MVDR magnitude squared coherence (MSC) analysis

As explained in Section 3.6, because of the shortness of the temperature data the best coherence test can be made by directly comparing the frequencies of the temperature spectrum with the theoretical astronomical frequencies, as reported in Table 1. However, magnitude squared coherence (MSC) analysis with $L=20$ and 30 year window was used by Holm (2014a) to claim that the expected coherence could not be found: see Figure 3C. Here I discuss the problems of Holm's analysis and show how MSC should be used for getting some reasonable results.

Here I calculate the magnitude squared coherence (MSC) analysis using the Capon's approach known as the minimum variance distortion-less response (MVDR) method (Benesty et al., 2006). The MVDR-MSC method is chosen because it is a high-resolution method of coherence analysis. It provides much sharper and reliable results than the one based on the popular Welch's method implemented in the MATLAB function mscohere.m used in Holm (2014a) and reported here in Figure 3C. The MVDR-MSC method produces sharp peaks approximately like MEM and it is more appropriate to separate close frequencies using short windows. MVDR-MSC is based on the evaluation of the following MSC equation:

$$
\gamma_{x y}^{2}(\omega)=\frac{\left|S_{x y}(\omega)\right|^{2}}{S_{x x}(\omega) S_{y y}(\omega)}=\frac{\left|\mathbf{f}^{H} \mathbf{R}_{x x}^{-1} \mathbf{R}_{x y} \mathbf{R}_{y y}^{-1} \mathbf{f}\right|^{2}}{\left[\mathbf{f}^{H} \mathbf{R}_{x x}^{-1} \mathbf{f}\right]\left[\mathbf{f}^{H} \mathbf{R}_{y y}^{-1} \mathbf{f}\right]}
$$



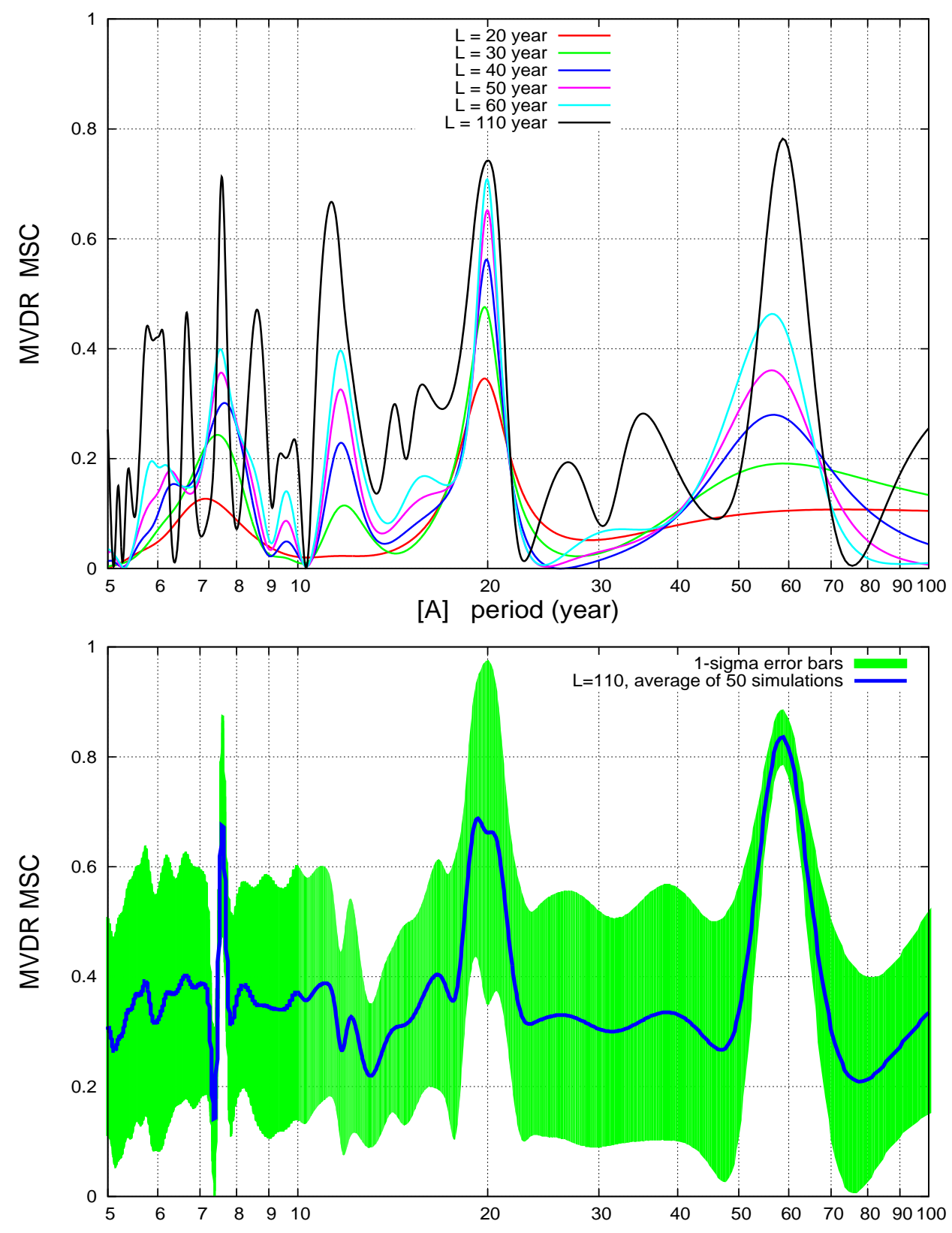

[B] period (year)

Fig. 10 [A] MVDR magnitude squared coherence (MSC) between the global surface temperature record and the Sun's speed relative to the barycenter of the solar system using Eq. 8 Different window lengths $L$ are used. The global surface temperature record is detrended of its quadratic fit to eliminate the non stationary upward bias, while the Sun's speed record is detrended only of its average value before applying the MVDR MSC algorithm (Benesty et al., 2006). [B] Montecarlo confidence test. It shows the average of 50 MVDR-MSC simulations between the astronomical record and the synthetic records produced with Eq.9 MVDR-MSC peaks larger than 0.6-0.7 are statistically significant.

where $S(\omega)$ is the cross-spectrum and $\mathbf{R}$ is the cross-correlation $(L \times L)$ matrix between the input time series $x(t)$ and $y(t)$, and $\mathbf{f}$ is a vector made of the harmonics of $\omega, f_{j}(\omega)=e^{i \omega j} / \sqrt{L}$, with $j=0,1, \ldots L-1$, where $L$ is the window length. By mathematical construction $0 \leq \gamma_{x y}^{2}(\omega) \leq 1$, and $\gamma_{x y}^{2}(\omega)$ theoretically approaches 1 if the two original sequences present a common major harmonic at the frequency $\omega$. See Benesty et al. (2006) for details and computer simulations that demonstrate the superiority of this methodology over the mscohere.m algorithm. 
Figure 10A shows the MVDR-MSC using $L=20$ and 30 year (I added the curves for 40, 50 and 60 years because also tested but not shown by Holm), and $L=110$ years that should be chosen to better solve the close major harmonics that can be detected in the 164-year temperature record. Figure 10A clearly shows that by increasing the window length to $L=110$ years a larger number of expected coherence peaks become manifest. Four major coherence peaks $\left(\gamma_{x y}^{2}(\omega) \gtrsim 0.7\right)$ appear at about 7.5, 11, 20 and 60 year periods. Minor coherence peaks at $\left(0.4<\gamma_{x y}^{2}(\omega)<0.5\right)$ may exist also at about 6 , 6.7 and 8.7 periods, but this result is less certain.

Note that a sharp coherence peak within the range 8.85-9.30 year is not supposed to be seen in Figure 10A because this period refers to lunar harmonics not present in the Sun's speed. If the temperature oscillations present a small degree of non-linearity and chaotic variation, as reasonable, its MSC values would be smaller than the case of perfectly harmonic oscillations.

The result depicted in Figure 10A confirms what could be deduced visually from Figures 5 and 7 (see Table 1) and Figure 8, and contradicts the results of Holm (2014a) (depicted here in Figure 3C) who concluded that only a weak "coherence at a period of 15-17 years can be found in the data." Indeed, Holm used a MSC methodology with windows of length $L=20$ years and $L=30$ years, which are even shorter than the already inadequate $L=60$ year window length used for his time-frequency analysis: their spectral resolution is $\nabla f_{20}=1 / 20=0.05 \mathrm{yr}^{-1}$ and $\nabla f_{30}=1 / 30=0.033$ $y^{-1}$. Moreover, the function mscohere.m used in Holm produces large lobes and does not separate well close frequencies (Benesty et al., 2006).

Benesty et al. (2006) gives no statistical properties for the MVDR-MSC estimate. To test the significance of the coherence spectral peaks shown in Figure 10A, a Montecarlo test is performed. The astronomical record, whose harmonic dynamics is well defined and the record is highly accurate, is processed against an ensemble of synthetic temperature signals made of the three proposed coherence harmonics of the type:

$$
g(t)=\cos \left(\frac{2 \pi t}{7.5+0.17 \vartheta}+\phi\right)+1.7 \cos \left(\frac{2 \pi t}{20+1.2 \vartheta}+\phi\right)+4.3 \cos \left(\frac{2 \pi t}{60+5 \vartheta}+\phi\right)+\xi_{t}
$$

where $\phi, \vartheta$ and $\xi_{t}$ are Gaussian normal random noise. The relative amplitudes of Eq. 9 were deduced from Figure 7B in units of the smallest one among the three. The periods were made to vary within a reasonable spectral error deduced from Table 1 and Figure 7B to simulate possible nonlinear and chaotic climatic responses to the hypothesized external harmonic forcing. The purpose of the test was to study the statistics of the three expected coherent MVDR-MSC peaks.

Fifty simulations were run. Their average and standard deviation band are shown in Figure 10B. The simulation produces the expected coherence peaks at 7.5, 20 and 60 year periods with average MVDR-MSC values between 0.65 and 0.85 , which is a range compatible with the peak values observed in Figure 10A. Thus, limited to the three chosen harmonics and relative to the MVDR-MSC measure, the temperature signal is statistically equivalent to the harmonic model of Eq. 9 . Figure 10B also shows that using a 164-year monthly record MVDR-MSC values smaller than 0.6 would not be distinguishable from noise.

Finally, the inadequacy of Holm's analysis can be easily demonstrated using computer generated sequences. I generated two artificial sequences of 164 data points, like the 164-year temperature signal, of the type 

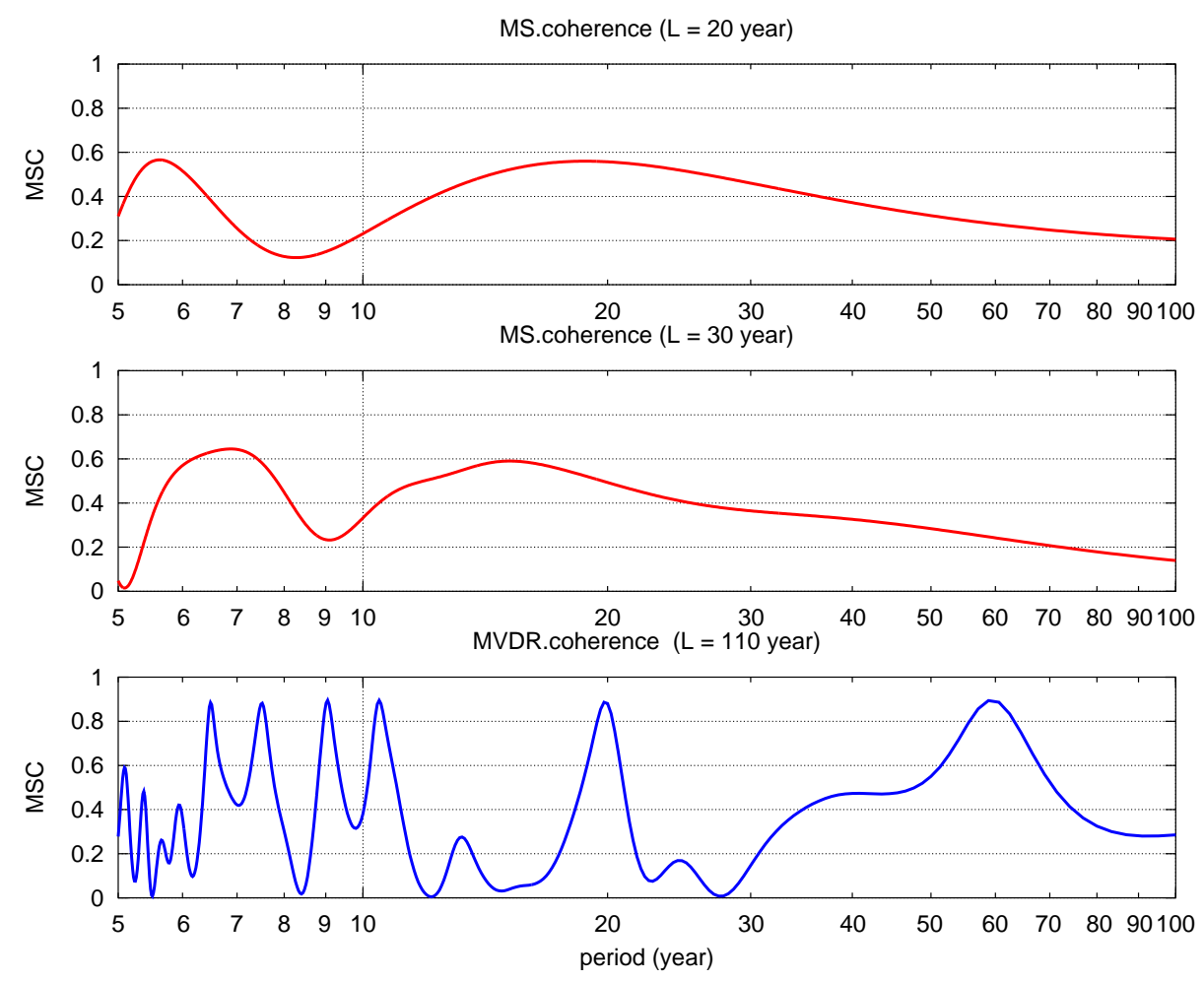

Fig. 11 Typical magnitude squared coherence (MSC) between two computer generated signals made of 6 harmonics plus random noise (Eq. 10. The upper two panels use the MATLAB function ms cohere .m adopted in Holm (2014a) with windows of $L=20$ and 30 years (red curves). The bottom panel use the MVDR coherence method with $L=110$ years (blue curves). Only in the latter case the 6 theoretical frequencies $\left(\Omega_{1}=1 / 60 \mathrm{yr}^{-1} ; \Omega_{2}=1 / 20 \mathrm{yr}^{-1} ; \Omega_{3}=1 / 10.4 \mathrm{yr}^{-1} ; \Omega_{4}=1 / 9.1 \mathrm{yr}^{-1} ; \Omega_{5}=1 / 7.5 \mathrm{yr}^{-1} ; \Omega_{6}=1 / 6.5 \mathrm{yr}^{-1}\right)$ are well detected.

$$
f(t)=\sum_{i=1}^{6} \cos \left(2 \pi \Omega_{i} t+\phi_{i}\right)+\xi_{t}
$$

where $\phi_{i}$ are random phases, $\xi_{t}$ is Gaussian normal random noise, and the frequencies $\Omega_{i}$ are similar to those found in the temperature record: $\Omega_{1}=1 / 60 \mathrm{yr}^{-1} ; \Omega_{2}=1 / 20 \mathrm{yr}^{-1} ; \Omega_{3}=1 / 10.4 \mathrm{yr}^{-1} ; \Omega_{4}=1 / 9.1 \mathrm{yr}^{-1} ; \Omega_{5}=1 / 7.5 \mathrm{yr}^{-1}$; $\Omega_{6}=1 / 6.5 \mathrm{yr}^{-1}$. Then I calculated the MSC between the two generated signals using the MATLAB function mscohere.m as used in Holm (2014a) with windows of $L=20$ and 30 years, and I repeat the analysis of the same computer generated records using the MVDR MSC method with $L=110$ years. The results are depicted in Figure 11. Only in the low panel case (blue curve), which uses the MVDR MSC method, the 6 common frequencies are clearly detected. On the contrary, the two upper panels show vague mscohere curves made of two wide lobes within the 5-10 and 10-20 year period bands: this is the same pattern shown in Holm's figures 4 and 5 (his figure 5 is shown here in Figure 3C). Thus, the evidence is that Holm's MSC methodology fails to identify close harmonics similar to those that need to be searched and, therefore, it cannot be used to test whether or not the temperature signal contains astronomical harmonics.

In conclusion, Holm's analysis, by using too short spectral windows and a poor MSC algorithm, is corrupted by too large frequency error bars that within the analyzed scales cover contiguous expected astronomical peaks. This is suggested by the two wide spectral lobes shown in Figure 3C, which are reproduced in the computer experiment depicted in Figure 11. With short $L=20$ and 30 year windows the mscohere.m algorithm simply clusters various contiguous 
harmonics into two average groups, one between 5 and 10 years and the other between 10 and 20 years; the 60-year coherence cannot be detected at all because such a period simply does not enter within a 20 or 30 year window. Nevertheless, Holm plotted his coherence graphs (e.g. Figure 3C) up to periods of 100 years giving the misleading impression that there is no spectral coherence at the 60 -year period.

\section{Conclusion}

Since 1859, when Wolf proposed that solar variability could be modulated by the combined effect of the planets, solar scientists have been wondering about the possibility of a planetary theory of solar variation, which would also imply a planetary theory of climate change. Numerous papers have recently appeared in the scientific literature supporting such a theory using empirical analysis of data and proposing models that attempt to interpret the data patterns with some combinations of planetary harmonics (e.g.: Abreu et al., 2012; Jakubcová and Pick, 1986; McCracken et al., 2014, 2013; Mörner et al., 2013; Puetz et al., 2014; Salvador, 2013; Scafetta, 2010, 2012d, 2013a, b, 2014; Scafetta and Willson, 2013b)a, 2014; Tan and Cheng, 2013; Wilson, 2013, and many others). However, some critiques have also appeared questioning the empirical findings.

I have shown that Cauquoin et al. (2014) used interesting data and analysis but they misinterpreted the complexity of the planetary theory of solar variation because they focused only on the planetary model proposed by Abreu et al. (2012) and did not highlight some important details that their analysis was revealing. I have shown that Cauquoin et al. (2014)'s own analysis of high-resolution cosmogenic solar activity proxy records both during the Holocene and during the Marine Interglacial Stage 9.3 (MIS 9.3), 325-336 kyear ago reveals that these two independent solar proxy records show four common spectral peaks at about 103, 115, 130 and 150 years at about the 95\% or above confidence level. These are the typical 100-150 year oscillations that regulate the alternating of grand solar minima and maxima similar to the Wolf, Spörer, Maunder and Dalton grand solar minima observed during the last centuries. I have shown that the planetary model proposed by Scafetta (2012c) predicts these four harmonics well. The peculiarity of my model over that proposed by Abreu et al. (2012) is that in my model solar variation is due to a modulation of the Schwabe 11-year solar cycle by planetary harmonic tidal forcings plus a reasonable non-linear response of the internal solar mechanisms to the external planetary harmonic forcings. Therefore, solar variation depends on a coupling between external and internal solar mechanisms. This model may be physically more plausible to explain some of the typical observed harmonics found in the solar proxy models (cf. Ogurtsov et al., 2002).

On the decadal and multidecadal scale Holm (2014a) claimed that time-frequency analysis based on $L=60$ year window and magnitude squared coherence (MSC) analysis based on $L=20$ and 30 year window would demonstrate that global surface temperature data do not contain time-invariant spectral lines nor frequencies coherent to astronomical oscillations. He questioned Scafetta (2010) where it was first proposed that the climate system contains a signature of multiple astronomical harmonics of solar, lunar and planetary origin. I have demonstrated that Holm (2014a) conclusion is flawed because based on a misapplication of the time-frequency analysis methodology that also yield astronomical paradoxes. Essentially, the windows lengths used in Holm (2014a) are too short to separate the frequencies of interest. 
Using window lengths of $L=60$ years for the time frequency analysis, the spectral resolution of his analysis is $\nabla f_{60}=$ $1 / 60=0.0167 \mathrm{yr}^{-1}$; using window lengths of $L=20$ and 30 year for the magnitude squared coherence analysis, the spectral resolution of his analysis is $\nabla f_{20}=1 / 20=0.05 \mathrm{yr}^{-1}$ and $\nabla f_{30}=1 / 30=0.033 \mathrm{yr}^{-1}$, respectively. However, the theoretical spectral resolution required for separating the astronomical expected harmonics is $\nabla f \leq 1 / 178.34=0.0056$ $y r^{-1}$, as seen in Figure 5B. Therefore, Holm's methodologies did not have the required spectral resolution to do the job and could not test whether or not the global surface temperature contains a signature of astronomical frequencies.

I have demonstrated that only a minimal windows of $L \gtrsim 178.4$ years can theoretically well solve the beats among the expected major astronomical frequencies, but the temperature record is only 164 years long. Therefore, the best analysis that can be done right now is to use only one 164-year window covering the entire available record as done in Scafetta (2010) and repeated here in Table 1 by directly comparing the spectra of Figures 5 and 7B with their confidence error. Table 1 shows that a spectral coherence among the global surface temperature and the main astronomical expected oscillations is clearly detected.

However, even if partially, a minimal window length of $L=110$ years can solve at least the major expected harmonics. Using $L=110$ year both the time-frequency analysis and the magnitude squared coherence analysis clearly reveal the spectral coherence among the main global surface temperature and the astronomical oscillations that can be detected using the available 164-year long global surface temperature sequences, as shown in Figures 8 and 10. Finally, Figures 6 and 11 demonstrate the inadequacy of Holm's analysis with simple computer experiments.

Indeed, several climatic oscillations have been observed throughout the Holocene and can be easily associated with solar, planetary and lunar harmonics as discussed in the Introduction.

In conclusion, Cauquoin et al. (2014) is quite interesting but these authors misunderstood the complexity of the planetary theory of solar variation. On the contrary, Holm (2014a)'s results are definitely artifacts of his inadequate window size used in both his time frequency analysis and magnitude squared coherence analysis, which was even worsen by the low quality mscohere.m algorithm that he used: essentially, his results are misleading.

\section{Appendix}

A) The three frequency solar model

Here I summarize the functions used for constructing the planetary/solar three frequency solar model discussed in Section 2 and shown in Figure 2. This appendix reproduces the Appendix in Scafetta (2012c) for the reader convenience.

The three basic proposed harmonics are:

$$
\begin{aligned}
& h_{1}(t)=0.83 \cos \left(2 \pi \frac{t-2000.475}{9.929656}\right) \\
& h_{2}(t)=1.0 \cos \left(2 \pi \frac{t-2002.364}{10.87}\right)
\end{aligned}
$$




$$
h_{3}(t)=0.55 \cos \left(2 \pi \frac{t-1999.381}{11.862242}\right)
$$

where the relative amplitudes are weighted on the sunspot number record since 1749. Three frequencies derive from the spectrum of the sunspot record (see Figure 2A) where the two side harmonics at 9.93 and 11.86 year period are theoretically deduced from the tidal oscillations generated by Jupiter and Saturn. The three phases are deduced: from the conjunction date of Jupiter and Saturn, $t=2000.475$; the perihelion date of Jupiter, $t=1999.381$; and by regression on the sunspot cycle, $t=2002.634$.

The basic harmonic model is

$$
\begin{aligned}
& h_{123}(t)=h_{1}(t)+h_{2}(t)+h_{3}(t) \\
& f_{123}(t)=h_{123}(t) \quad \text { if } \quad h_{123}(t) \geq 0 \\
& f_{123}(t)=0 \quad \text { if } \quad h_{123}(t)<0
\end{aligned}
$$

which is depicted in Figure 2B.

The chosen beat function modulations in generic relative units and their sum are:

$$
\begin{aligned}
& b_{12}(t)=0.60 \cos \left(2 \pi \frac{t-1980.528}{114.783}\right) \\
& b_{13}(t)=0.40 \cos \left(2 \pi \frac{t-2067.044}{60.9484}\right) \\
& b_{23}(t)=0.45 \cos \left(2 \pi \frac{t-2035.043}{129.951}\right) \\
& b_{123}(t)=b_{12}(t)+b_{13}(t)+b_{23}(t)+1 .
\end{aligned}
$$

The three relative amplitudes are roughly estimated against Eq. 15. The millennial modulating function is

$$
g_{m}(t)=A \cos \left(2 \pi \frac{t-2059.686}{983.401}\right)+B
$$

The parameters $A$ and $B$ may be changed according to the application. The two proposed modulated solar/planetary functions are

$$
\begin{gathered}
F_{123}(t)=g_{m}(t) f_{123}(t) \text { with } A=0.2, B=0.8 \\
B_{123}(t)=g_{m}(t) b_{123}(t) \text { with } A=0.3, B=0.7 .
\end{gathered}
$$


See Scafetta (2012c) for more details and for a supplement file with the actual data.

B) Scafetta (2010) astronomical - temperature spectral coherence

For convenience of the reader Figure 12 reproduces figure 6B and 9A of Scafetta (2010). Figure 12B shows MEM evaluations of numerous climatic records such as the global surface temperature $(G)$, the northern and southern global surface temperatures (GN and GS), the global, northern and southern land surface temperatures (L, LN, LS) and the global, northern and southern ocean surface temperatures ( $\mathrm{O}, \mathrm{ON}, \mathrm{OS})$. The green bars are the main solar, astronomical and lunar expected harmonics (cf. Figure 5). It is easy to notice a coherence between the astronomical harmonics and the MEM spectral peaks at multiple frequencies. Figure 12A directly compares the temperature average periods (red) against the astronomical average periods (blue). A $\chi^{2}$ test output among the various frequencies is shown suggesting that the coherence confidence is at the $96 \%$. Additional calculations and evidences are provided in Scafetta (2010).

C) Kepler's diagram of Jupiter-Saturn conjunctions

Figure 13 shows the original diagram of Jupiter-Saturn conjunctions prepared by Kepler (1606). It highlights the date and the constellation position of the great conjunctions, that occur every 20 years, from 1583 to 1763 . The 60-year trigon pattern, that involves three consecutive conjunctions, is clearly visible together with its slow millennial rotation. The 20, 60 and 800-1000 year oscillations associated to the movement of Jupiter and Saturn were well known since antiquity and used to construct some kind of astrological-based climate models (Kepler, 1606; Iyengar, 2009; Ma'sar, 886; Temple, 1998). See Scafetta (2012a) for additional details. 

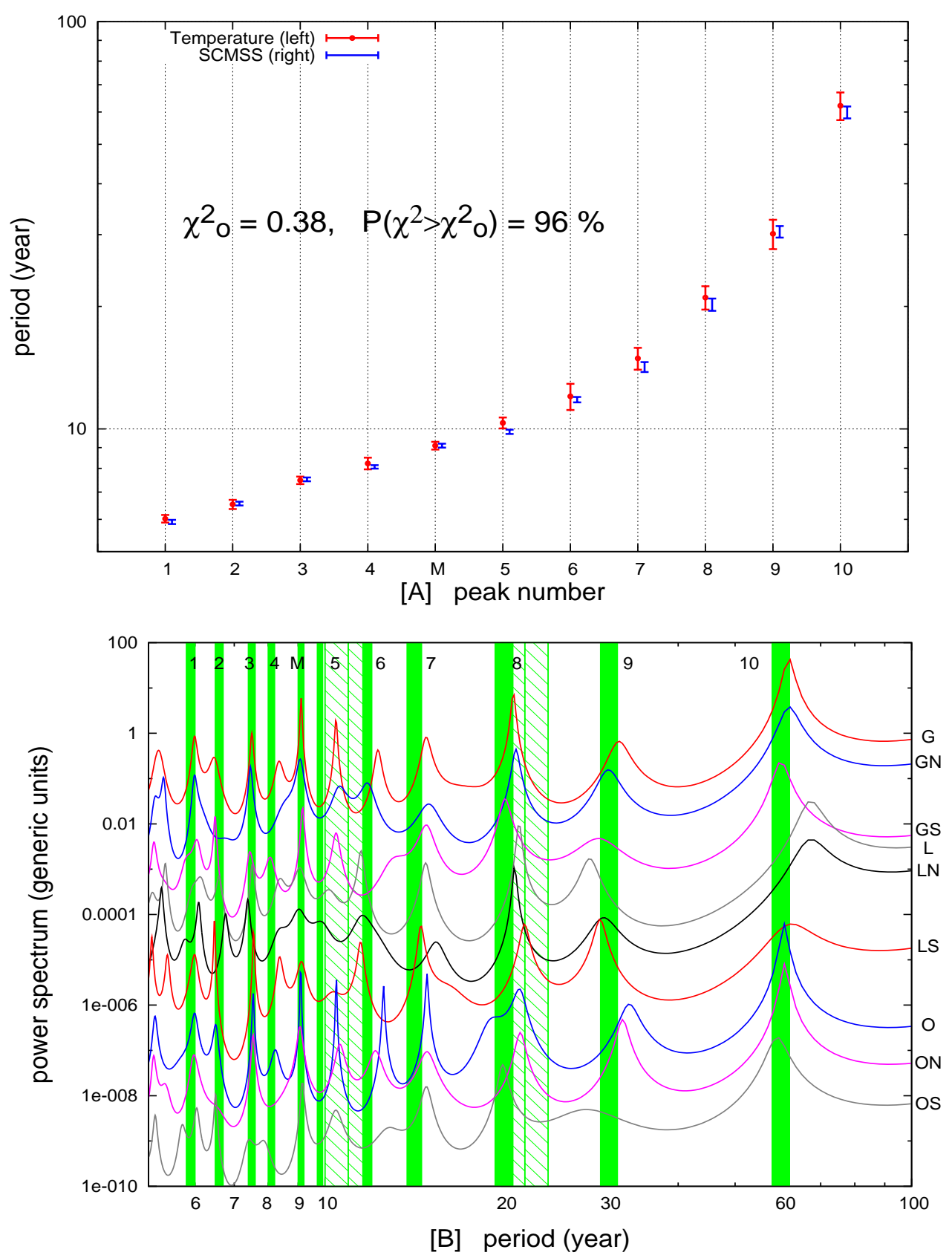

Fig. 12 Reproduction of figure 6B and 9A of Scafetta (2010) showing [A] the $\chi^{2}$ spectral coherence test and [B] the direct comparison between the MEM curve of several climatic records and the astronomical, solar and lunar harmonics (green bars). See the original paper for details and addition.

\section{References}

Abreu, J. A., Beer, J., Ferriz-Mas, A., McCracken, K. G., Steinhilber, F., 2012. Is there a planetary influence on solar activity? Astron. Astrophys., 548, A88.

Agnihotri, R., Dutta, K., 2003. Centennial scale variations in monsoonal rainfall (Indian, east equatorial and Chinese monsoons): manifestations of solar variability. Curr. Sci., 85, 459-463. 


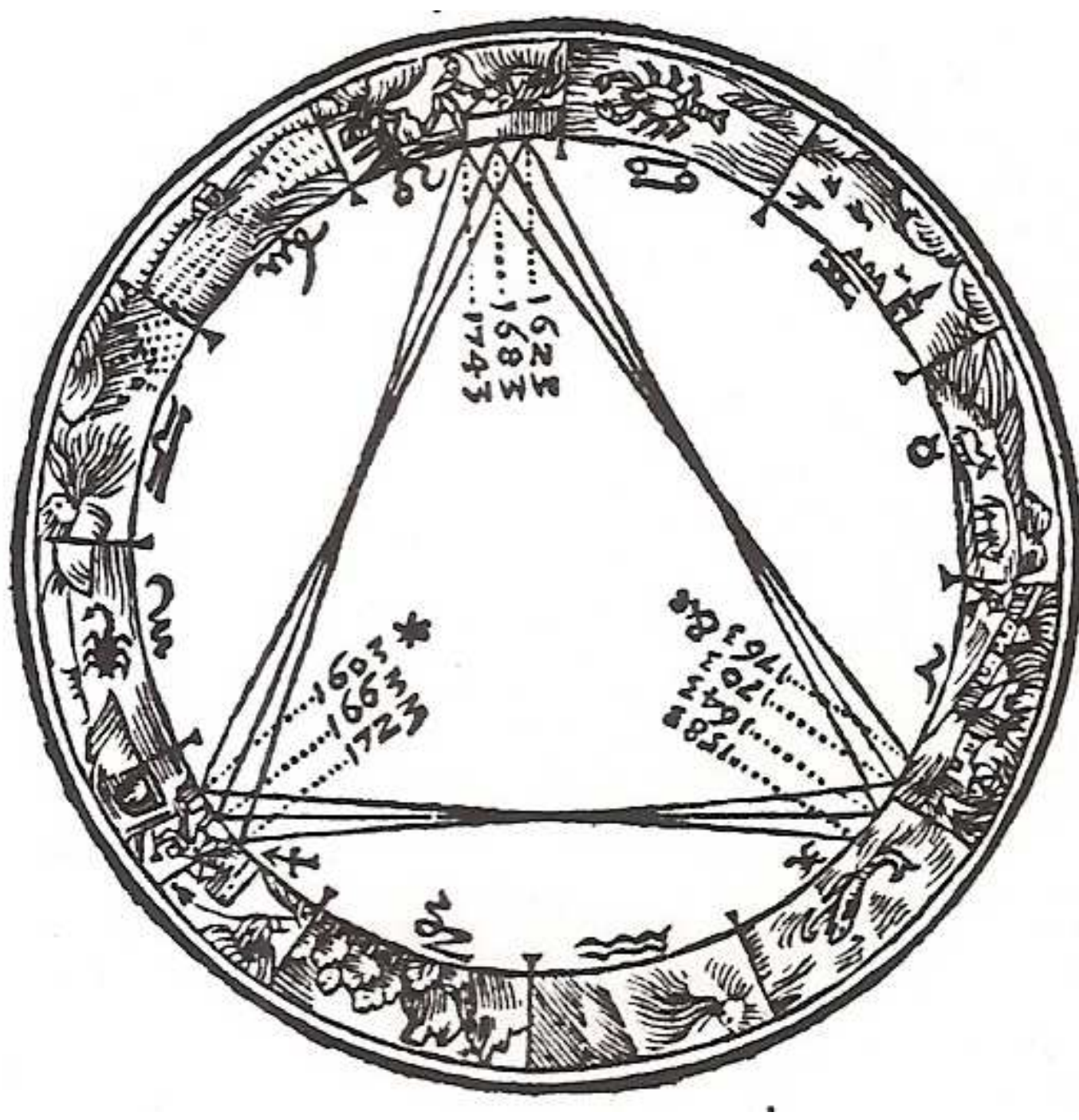

Fig. 13 The original diagram of Jupiter-Saturn conjunctions prepared by Kepler (1606)

Bard, E., Raisbeck, G., Yiou, F., Jouzel, J., 2000. Solar irradiance during the last 1200 years based on cosmogenic nuclides. Tellus, 52B, 985-992.

Benesty, J., Chen, J., Huang, Y., 2006. Estimation of the coherence function with the MVDR approach. Acoustics, Speech and Signal Processing. ICASSP 2006 Proceedings 3, 500-503. doi: 10.1109/ICASSP.2006.1660700. http://www.mathworks.com/matlabcentral/fileexchange/9781-coherence-function/content/coherence_MVDR

Bennett, J., Donahue, M., Schneider, N., Voit, M., 2014. The cosmic perspective (seventh edition). (Pearson Education, San Francisco CA).

Bond, G., Kromer, B., Beer, J., Muscheler, R., Evans, M.N., Showers,W., Hoffmann, S., Lotti- Bond, R., Hajdas, I., Bonani, G., 2001. Persistent solar influence on North Atlantic climate during the Holocene. Science, 294, 2130-2136.

Brohan, P., Kennedy, J. J., Harris, I., Tett, S. F. B., Jones, P. D., 2006. Uncertainty estimates in regional and global observed temperature changes: a new dataset from 1850. J. Geophys. Res., 111, D12106. doi:10.1029/ 2005JD006548.

Callebaut, D. K., de Jager, C., Duhau, S., 2012. The influence of planetary attractions on the solar tachocline. J. Atmos. Solar Terr. Phys., 80, 73-78. 
Cauquoin, A., Raisbeck, G. M., Jouzel, J., Bard, E., and ASTER Team, 2014. No evidence for planetary influence on solar activity 330000 years ago. Astron. Astrophys., 561, A132.

Charvátová, I., 2009. Long-term predictive assessments of solar and geomagnetic activities made on the basis of the close similarity between the solar inertial motions in the intervals 1840-1905 and 1980-2045. New Astron., 14, 25-30.

Christiansen, B., Ljungqvist, F. C., 2012. The extra-tropical Northern Hemisphere temperature in the last two millennia: reconstructions of low-frequency variability. Clim. Past, 8, 765-786.

Chylek, P., Folland, C. K., Dijkstra, H. A., Lesins, G., Dubey, M. K., 2011. Ice-core data evidence for a prominent near 20 year time-scale of the Atlantic Multidecadal Oscillation. Geophys. Res. Lett., 38, L13704.

Copernicus, N., 1543. De revolutionibus orbium coelestium. (Johannes Petreius).

Davis, J. C., Bohling, G., 2001. The search for patterns in ice-core temperature curves. In: Gerhard, L.C., Harrison, W.E., Hanson, B.M. (Eds.), Geological Perspectives of Global Climate Change, pp. 213-229.

Fairbridge, R.W. and Shirley, J. H., 1987. Prolonged minima and the 179- year cycle of the solar inertial motion. Sol. Phys., $10,191-210$.

FFT Fundamentals: http://zone.ni.com/reference/en-XX/help/372416A-01/svtconcepts/fft_funda/

Geddes, A. B. and King-Hele, D. G., 1983. Equations for Mirror Symmetries Among the Distances of the Planets. Q. J. R. Astron. Soc., 24, 10-13.

Ghil, M., Allen, R. M., Dettinger, M. D., Ide, K., Kondrashov, D., Mann, M. E., Robertson, A., Saunders, A., Tian, Y., Varadi, F., Yiou, P., 2002. Advanced spectral methods for climatic time series. Rev. Geophys., 40, 3.1-3.41 (SSA-MTM tool kit for spectral analysis).

Grinsted, A., Moore, J. C., Jevrejeva, S., 2004. Application of the cross wavelet transform and wavelet coherence to geophysical time series. Nonlin. Proc. Geophys., 11, 561-566

Goldreich, P. and Peale, S. J., 1966. Resonant Rotation for Venus? Nature, 209, 1117-1118.

Hoyt, D. V., Schatten, K. H., 1997. The Role of the Sun in the Climate Change. Oxford Univ. Press, New York.

Holm, S., 2014a. On the alleged coherence between the global temperature and the Sun's movement. J. Atmos. Solar Terr. Phys., 110-111, 23-27.

Holm, S., 2014b. Corrigendum to "On the alleged coherence between the global temperature and the sun's movement". http://arxiv.org/abs/1307.1086 [v3, Wed, 23 Apr 2014 13:14:40 GMT]. J. Atmos and Sol.-Terr. Phys. 119 230-231 (2014b).

Hung, C.-C.: Apparent Relations Between Solar Activity and Solar Tides Caused by the Planets, NASA report/TM2007-214817, available at: http:/ /ntrs.nasa.gov/search.jsp?R= 20070025111, 2007.

Iyengar, R. N., 2009. Monsoon rainfall cycles as depicted in ancient Sanskrit texts. Curr. Sci. 97, 444-447.

Jakubcová, I. and Pick, M., 1986. The planetary system and solarterrestrial phenomena. Stud. Geophys. Geod., 30, 224-235.

Jevrejeva, S., Moore, J. C., Grinsted, A., Woodworth, P., 2008. Recent global sea level acceleration started over 200 years ago? Geophys. Res. Lett., 35, L08715.

Jose, P. D., 1965. Sun's motion and sunspots. Astron. J., 70, 193-200. 
Kepler, J., 1596. Mysterium Cosmographicum (The Cosmographic Mystery), 1596.

Kepler, J., 1606. De Stella Nova in Pede Serpentarii (On the new star in Ophiuchus's foot).

Kerr, R. A., 2001. A variable sun paces millennial climate. Science, 294, 1431-1433.

Klyashtorin, L. B., Borisov, V., Lyubushin, A., 2009. Cyclic changes of climate and major commercial stocks of the Barents Sea. Mar. Biol. Res., 5, 4-17.

Knudsen, M. F., Seidenkrantz, M.-S., Jacobsen, B. H., Kuijpers, A., 2011. Tracking the Atlantic Multidecadal Oscillation through the last 8,000 years. Nature Commun., 2, 178.

Loehle, C., Scafetta, N., 2011. Climate change attribution using empirical decomposition of climatic data. Open Atmos. Sci. J., 5, 74-86.

Manzi, V., Gennari, R., Lugli, S., Roveri, M., Scafetta, N., Schreiber, C., 2012. High-frequency cyclicity in the Mediterranean Messinian evaporites: evidence for solar-lunar climate forcing. J. Sed. Res., 82, 991-1005.

Ma'sar, A., 886. In: Yamamoto, K., Burnett, C. (Eds.), On Historical Astrology-The Book of Religions and Dynasties (On the Great Conjunctions). Brill Academic Pub (January 1, 2000).

Mazzarella, A., Scafetta, N., 2012. Evidences for a quasi 60-year North Atlantic Oscillation since 1700 and its meaning for global climate change. Theor. Appl. Climatol., 107 (3-4), 599-609.

McCracken, K. G., Beer, J., Steinhilber, F., Abreu J., 2013. A Phenomenological Study of the Cosmic Ray Variations over the Past 9400 Years, and Their Implications Regarding Solar Activity and the Solar Dynamo. Sol. Phys., 286(2), 609-627.

McCracken, K. G., Beer, J., Steinhilber, F., Abreu J., 2014. Evidence for Planetary Forcing of the Cosmic Ray Intensity and Solar Activity Throughout the Past 9400 Years. Sol. Phys., 286(2), 609-627. Doi: 10.1007/s11207-014-0510-1

Molchanov, A. M., 1968. The Resonant Structure of the Solar System: The Law of Planetary Distances. Icarus, 8, $203-215$.

Molchanov, A. M., 1969a. The Reality of Resonances in the Solar System. Icarus, 11, 104-110.

Molchanov, A. M., 1969b. Resonances in Complex Systems: A Reply to Critiques. Icarus, 11, 95-103.

Mörner, N.-A., Tattersall, R., Solheim, J.-E., 2013. Preface: Pattern in solar variability, their planetary origin and terrestrial impacts. Pattern Recogn. Phys., 1, 203-204, doi:10.5194/prp-1-203-2013 http://www.pattern-recogn-phys.net/special_issue2.html

Ogurtsov, M. G., Nagovitsyn, Y. A., Kocharov, G. E., Jungner, H., 2002. Long-period cycles of the sun's activity recorded in direct solar data and proxies. Sol. Phys., 211, 371-394.

Pikovsky, A., Rosenblum, M., Kurths, J., 2001. Synchronization, a universal concept in nonlinear science, Cambridge University Press, Cambridge, UK.

Press,W. H., Teukolsky, S. A., Vetterling,W. T., and Flannery, B. P., 1997. Numerical Recipes in C, 2nd Edn., Cambridge University Press.

Poluianov, A., Usoskin, I., 2014. Critical Analysis of a Hypothesis of the Planetary Tidal Influence on Solar Activity. Sol. Phys., 289, 2333-2342.

Puetz, S. J., Prokoph, A., Borchardt, G, Mason, E. W., 2014. Evidence of synchronous, decadal to billion year cycles in geological, genetic, and astronomical events. Chaos, Solitons \& Fractals, 62-63, 55-75. 
Pustil'nik, L. A., Din G. Y., 2004. Influence of solar activity on the state of the wheat market in medieval England. Sol. Phys., 223, 335-356.

Qian, W.-H., Lu, B., 2010. Periodic oscillations in millennial global-mean temperature and their causes. Chin. Sci. Bull., $55,4052-4057$.

Salvador, R. J., 2013 A mathematical model of the sunspot cycle for the past 1000 year. Pattern Recogn. Phys., 1, 117-122. Scafetta N., Grigolini, P., Imholt, T., Roberts, J. A., West, B. J., 2004. Solar turbulence in earth's global and regional temperature anomalies. Phys. Rev. E, 69, 026303. DOI: 10.1103/PhysRevE.69.026303

Scafetta, N., 2010. Empirical evidence for a celestial origin of the climate oscillations and its implications. J. Atmos. Solar Terr. Phys., 72 (13), 951-970.

Scafetta, N., 2012a. A shared frequency set between the historical mid-latitude aurora records and the global surface temperature. J. Atmos. Solar Terr. Phys., 74, 145-163.

Scafetta, N., 2012b. Testing an astronomically based decadal-scale empirical harmonic climate model versus the IPCC (2007) general circulation climate models. J. Atmos. Solar Terr. Phys., 80, 124-137.

Scafetta, N., 2012c. Multi-scale harmonic model for solar and climate cyclical variation throughout the holocene based on Jupiter-Saturn tidal frequencies plus the 11-year solar dynamo cycle. J. Atmos. Solar Terr. Phys., 80, $296-311$.

Scafetta N., 2012d. Does the Sun work as a nuclear fusion amplifier of planetary tidal forcing? A proposal for a physical mechanism based on the mass-luminosity relation. J. Atmos. Solar Terr. Phys., 81-82, 27-40.

Scafetta N., 2013a. Solar and planetary oscillation control on climate change: hind-cast, forecast and a comparison with the CMIP5 GCMs. Energy \& Environment, 24(3-4), 455-496.

Scafetta, N., 2013b. Discussion on climate oscillations: CMIP5 general circulation models versus a semi-empirical harmonic model based on astronomical cycles. Earth Sci. Rev., 126, 321-357.

Scafetta, N., 2014. The complex planetary synchronization structure of the solar system. Pattern Recogn. Phys., 2, 1-19. DOI: $10.5194 /$ prp-2-1-2014.

Scafetta N., Humlum, O., Solheim, J.-E., and Stordahl, K., 2013. Comment on “The influence of planetary attractions on the solar tachocline" by Callebaut, de Jager and Duhau. J. Atmos. Solar Terr. Phys., 102, 368-371.

Scafetta N., Willson, R. C., 2013a. Planetary harmonics in the historical Hungarian aurora record (1523-1960). Planet. Space Sci., 78, 38-44.

Scafetta N., Willson, R. C., 2013b. Empirical evidences for a planetary modulation of total solar irradiance and the TSI signature of the 1.09-year Earth-Jupiter conjunction cycle. Astrophys. Space Sci., 348(1), 25-39.

Scafetta, N., and R. C. Willson, 2014. ACRIM total solar irradiance satellite composite validation versus TSI proxy models. Astrophys. Space Sci., 350(2), 421-442.

Scharf, C. A., 2010. Possible constraints on exoplanet magnetic field strengths from planet-star Interaction. Astrophys. J., 722, 1547-1555.

Schulz, M., Mudelsee, M., 2002. REDFIT: Estimating red-noise spectra directly from unevenly spaced paleoclimatic time series. Comput. Geosci., 28, 421-426. 
Sharp, G. J., 2013. Are Uranus \& Neptune Responsible for Solar Grand Minima and Solar Cycle Modulation?, Int. J. Astron. Astrophys., 3, 260-273.

Shirley, J. H., Sperber, K. R., and Fairbridge, R. W., 1990. Sun's internal motion and luminosity. Sol. Phys., 127, 379-392.

Shkolnik, E., Walker, G. A. H., Bohlender, D. A., 2003. Evidence for planet-induced chromospheric activity on HD 179949. Astrophys. J., 597, 1092-1096.

Shkolnik, E., Walker, G. A. H., Bohlender, D. A., Gu, P.-G., Kurster, M., 2005. Hot Jupiters and hot spots: The short- and long-term chromospheric activity on stars with giant planets. Astrophys. J., 622, 1075-1090.

Steinhilber, F., Abreu, J. A., Beer, J., Brunner, I., Christl, M., Fischer, H., Heikkila, U., Kubik, P. W., Mann, M., McCracken, K. G., Miller, H., Miyahara, H., Oerter, H., Wilhelms, F., 2012. 9,400 years of cosmic radiation and solar activity from ice cores and tree rings. PANAS, 109 (16), 5967-5971.

Tan, B., Cheng, Z., 2013. The mid-term and long-term solar quasiperiodic cycles and the possible relationship with planetary motions. Astrophys. Space Sci., 343, 511-521.

Tattersall, R., 2013. The Hum: log-normal distribution and planetary-solar resonance. Pattern Recogn. Phys., 1, 185-198, doi:10.5194/prp-1-185-2013.

Temple, R., 1998. The Sirius Mystery. (Destiny Books,). Appendix 3, "Why Sixty Years?". http://www.bibliotecapleyades.net/universo/siriusmystery/siriusmystery_appendix03.htm

Thejll, P., Lassen, K., 2000. Solar forcing of the northern hemisphere land air temperature: new data. J. Atmos. Solar Terr. Phys., 62, 1207-1213.

Titius, J. D., 1766. Betrachtung über die Natur, vom Herrn Karl Bonnet (Leipzig, 1766), pp. 7-8; transl. by Jaki, S., in: The early history of the Titius-Bode Law. Am. J. Phys., 40, 1014-1023, 1972.

Wang, Z., Wu, D., Song, X., Chen, X., Nicholls, S., 2012. Sun-moon gravitation-induced wave characteristics and climate variation. J. Geophys. Res., 117, D07102.

Wigley, T. M. L., 1988. The climate of the past 10,000 years and the role 366 of the Sun, in Secular Solar and Geomagnetic Variations in the Last 367 10,000 Years. (edited by F. R. Stephenson and A. W. Wolfendale, pp. 209-224, Springer, New York).

Wilson, I. R. G., 2013. The Venus-Earth-Jupiter spin-orbit coupling model. Pattern Recogn. Phys., 1, 147-158.

Wolf, R., 1859. Extract of a letter to Mr. Carrington. Mon. Not. R. Astron. Soc., 19, 85-86, 1859.

Wolff, C. L., Patrone, P. N., 2010. A new way that planets can affect the Sun. Sol. Phys., 266, 227-246.

Wright, J. T., Marcy, G. W., Butler, R. P., Vogt, S. S., Henry, G. W., Isaacson, H., Howard, A. W., 2008. The Jupiter Twin HD 154345b. Astrophys. J., 683(1), L63-L66. 